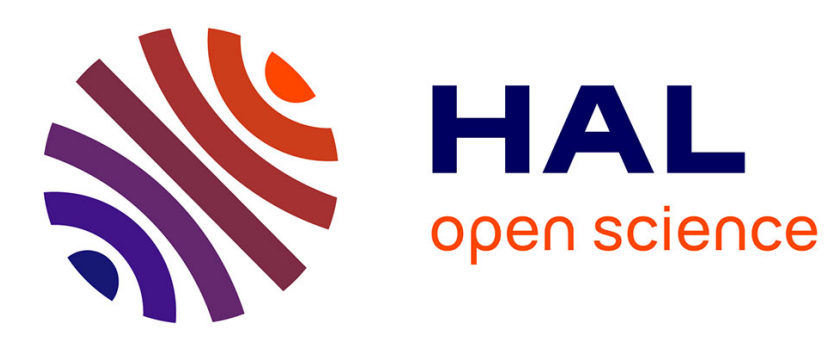

\title{
A low-frequency assumption for optimal time-decay estimates to the compressible Navier-Stokes equations
}

\author{
Jiang $\mathrm{Xu}$
}

\section{To cite this version:}

Jiang $\mathrm{Xu}$. A low-frequency assumption for optimal time-decay estimates to the compressible Navier-

Stokes equations. 2018. hal-01807632

\section{HAL Id: hal-01807632 \\ https://hal.science/hal-01807632}

Preprint submitted on 5 Jun 2018

HAL is a multi-disciplinary open access archive for the deposit and dissemination of scientific research documents, whether they are published or not. The documents may come from teaching and research institutions in France or abroad, or from public or private research centers.
L'archive ouverte pluridisciplinaire HAL, est destinée au dépôt et à la diffusion de documents scientifiques de niveau recherche, publiés ou non, émanant des établissements d'enseignement et de recherche français ou étrangers, des laboratoires publics ou privés. 


\title{
A LOW-FREQUENCY ASSUMPTION FOR OPTIMAL TIME-DECAY ESTIMATES TO THE COMPRESSIBLE NAVIER-STOKES EQUATIONS
}

\author{
JIANG XU
}

\begin{abstract}
The global existence issue in critical spaces for compressible NavierStokes equations, was addressed by Danchin (2000) and then developed by CharveDanchin (2010), Chen-Miao-Zhang (2010) and Hasport (2011) in more general $L^{p}$ setting. Our aim is to exhibit (more precisely) time-decay estimates of solutions constructed in the critical framework. As we known, the low-frequency assumption usually plays a key role in the large-time asymptotics of solutions, which was firstly observed in usual $L^{1}$ space by Matsumura and Nishida [30]. In this paper, we claim $a$ new low-frequency assumption for barotropic compressible Navier-Stokes equations, which may be of interest in the mathematical analysis of viscous fluids. Precisely, if the initial density and velocity additionally belong to some Besov space $\dot{B}_{2, \infty}^{-\sigma_{1}}$ with the regularity $\sigma_{1} \in(1-d / 2,2 d / p-d / 2]$, then a sharp time-weighted inequality including enough time-decay information can be available, where optimal decay exponents for the high frequencies are also exhibited. The proof mainly depends on tricky and non classical Besov estimates. As a by-product, those optimal time-decay rates of $L^{q}-L^{r}$ type are well captured in the critical regularity framework.
\end{abstract}

\section{INTRODUCTION}

Consider the following barotropic compressible Navier-Stokes equations

$$
\left\{\begin{array}{l}
\partial_{t} \varrho+\operatorname{div}(\varrho u)=0, \\
\partial_{t}(\varrho u)+\operatorname{div}(\varrho u \otimes u)-\operatorname{div}(2 \mu D(u)+\lambda \operatorname{div} u \mathrm{Id})+\nabla \Pi=0 .
\end{array}\right.
$$

Here, $u=u(t, x) \in \mathbb{R}^{d}$ (with $(t, x) \in \mathbb{R}_{+} \times \mathbb{R}^{d}(d \geq 2)$ ) and $\varrho=\varrho(t, x)$ stand for the velocity field and the density of fluid, respectively. The barotropic assumption means that the pressure $\Pi \triangleq P(\varrho)$ depends only upon the density and the function $P$ will be taken suitably smooth in all that follows. The notations div and $\nabla$ are the divergence operator and gradient operator with respect to $x$, respectively. $D(u) \triangleq \frac{1}{2}\left(D_{x} u+{ }^{T} D_{x} u\right)$ stands for the deformation tensor. The Lamé coefficients $\lambda$ and $\mu$ (the bulk and shear viscosities) are density-dependent functions, which are supposed to be smooth enough and to satisfy $\mu>0$ and $\nu \triangleq \lambda+2 \mu>0$.

System (1.1) is supplemented with the initial data

$$
\left.(\varrho, u)\right|_{t=0}=\left(\varrho_{0}(x), u_{0}(x)\right), x \in \mathbb{R}^{d},
$$

1991 Mathematics Subject Classification. 76N15, 35Q30, 35L65, 35K65.

Key words and phrases. Compressible Navier-Stokes system; time-decay estimates; critical Besov spaces.

The author would like to thank Professor A. Matsumura for introducing him to the decay problem for partially parabolic equations when he visited Osaka University. Also, he is grateful to the collaborator R. Danchin for addressing the conjecture on the regularity of low frequencies when visiting the LAMA in UPEC. 
and in this paper we focus on solutions that are close to some constant state $\left(\varrho_{\infty}, 0\right)$ with $\varrho_{\infty}>0$, at infinity.

So far there is a huge literature on the existence and large-time behavior of solutions to (1.1). The local existence and uniqueness of smooth solutions were proved by Serrin [34] and Nash [31]. The local existence of strong solutions with Sobolev regularity was constructed by Solonnikov [36], Valli [37] and Fiszdon-Zajaczkowski [12]. Matsumura and Nishida [29,30] established the first global strong solutions for small perturbations of a linearly stable constant state $\left(\varrho_{\infty}, 0\right)$ in three dimensions. With the additional $L^{1}$ assumption of initial data, the definite decay rate was also available:

$$
\left\|\left(\varrho-\varrho_{\infty}, u\right)(t)\right\|_{L^{2}} \lesssim\langle t\rangle^{-\frac{3}{4}} \quad \text { with }\langle t\rangle \triangleq \sqrt{1+t^{2}},
$$

which coincides with that of the heat kernel. Indeed, such time-decay rate reveals the dissipative properties of solutions to (1.1)-(1.2). Subsequently, Ponce [33] obtained more general $L^{p}$ decay rates:

$$
\left\|\nabla^{k}\left(\varrho-\varrho_{\infty}, u\right)(t)\right\|_{L^{p}} \lesssim\langle t\rangle^{-\frac{d}{2}\left(1-\frac{1}{p}\right)-\frac{k}{2}}, \quad 2 \leq p \leq \infty, \quad 0 \leq k \leq 2, \quad d=2,3 .
$$

S. Kawashima [23] exhibited the asymptotic behavior for generally hyperbolic-parabolic composite systems satisfying some dissipative assumption, which is now referred as the "Shizuta-Kawashima" stability condition. There are also some efforts extending Matsumura-Nishida's results to more physical situation where the fluid domain is not $\mathbb{R}^{d}$ : for example, the exterior domains were investigated by Kobayashi [26] and Kobayashi-Shibata [27], the half space by Kagei \& Kobayashi [24, 25]. In 1998, Xin [38] found a fact that any smooth solution to the Cauchy problem of full compressible Navier-Stokes system without heat conduction (including the present baratropic case) would blow up in finite time if the initial density contains vacuum. For the existence of solutions for arbitrary data, a breakthrough work is due to P.-L. Lions [21], where he obtained the global existence of weak solutions with finite energy when the adiabatic index is suitably large. Subsequently, some developments were made by Feireisl, Novotny \& Petzeltová [11] and Jiang-Zhang [19].

On the other hand, it is interesting to show the global existence of strong solutions in the critical regularity framework. More precisely, System (1.1) is invariant by the transformation

$$
\varrho(t, x) \rightsquigarrow \varrho\left(l^{2} t, l x\right), \quad u(t, x) \rightsquigarrow \ell u\left(l^{2} t, l x\right), \quad l>0
$$

up to a change of the pressure term $\Pi$ into $l^{2} \Pi$, hence one employs functional spaces to investigate (1.1), which are endowed with norms enjoying the scaling invariance (1.5). This trick is now classic and has been used by different authors. For instance, recall that the context of the incompressible Navier-Stokes equation, working in critical spaces goes back to the work by Fujita \& Kato in [13] (see also results by Kozono \& Yamazaki [28] and Cannone [2]). As observed by Danchin in [7], one may solve (1.1) globally in critical homogeneous Besov spaces of $L^{2}$ type, that is, the initial data belong to $\left(\dot{B}_{2,1}^{\frac{d}{2}} \cap \dot{B}_{2,1}^{\frac{d}{2}-1}\right) \times\left(\dot{B}_{2,1}^{\frac{d}{2}-1}\right)^{d}$. He developed an energy method on the mixed type system (1.1) and overcame the major difficulty that there is a loss of one derivative of density in the convection term. Later, his result has been extended to critical Besov spaces that are not related to $L^{2}$, by Charve-Danchin [4] and Chen-Miao-Zhang [6] independently. Recently, Haspot [15] achieved essentially the same result by using more elementary approach which is based on the use of Hoff's viscous effective flux [16] (see also [9] for the case of density-dependent viscosity coefficients). The reader is 
referred to $[4,6,15,9]$ or Theorem 1.1 in [10] directly. In this paper, our main interest is to exhibit (more precisely) the large time behavior of strong solutions constructed in the critical regularity framework. First of all, we would like to mention those efforts in this direction. Okita [32] studied the optimal time-decay estimates to (1.1)-(1.2) in the $L^{2}$ critical framework, thanks to a smart modification of the method of [7]. However, the result cannot cover the 2D case. In the survey paper [8], Danchin proposed another description of the time decay which allows to handle dimension $d \geq 2$. In the joint work with Danchin [10], we developed that method of [8] so as to establish the optimal decay rates in general $L^{p}$ critical spaces.

\section{Reformulation AND MAin RESUlts}

Next, in order to state main results of this paper, it is convenient to reformulate system (1.1) as the nonlinear perturbation form of constant equilibrium state $\left(\varrho_{\infty}, 0\right)$, looking at the nonlinearities as source terms. For simplicity, let's normalize the density $\varrho_{\infty}$, the sound speed $c_{\infty} \triangleq \sqrt{P^{\prime}\left(\varrho_{\infty}\right)}$ and the total viscosity $\nu_{\infty} \triangleq \lambda_{\infty}+2 \mu_{\infty}$ (with $\lambda_{\infty} \triangleq \lambda\left(\varrho_{\infty}\right)$ and $\left.\mu_{\infty} \triangleq \mu\left(\varrho_{\infty}\right)\right)$ to be one. Hence, we have

$$
\left\{\begin{array}{l}
\partial_{t} a+\operatorname{div} u=f \\
\partial_{t} u-\mathcal{A} u+\nabla a=g
\end{array}\right.
$$

with $f \triangleq-\operatorname{div}(a u), g \triangleq-u \cdot \nabla u-I(a) \mathcal{A} u-k(a) \nabla a+\frac{1}{1+a} \operatorname{div}(2 \widetilde{\mu}(a) D(u)+\widetilde{\lambda}(a) \operatorname{div} u \operatorname{Id}$, where

$$
\begin{gathered}
\mathcal{A} \triangleq \mu_{\infty} \Delta+\left(\lambda_{\infty}+\mu_{\infty}\right) \nabla \text { div such that } \nu_{\infty} \triangleq \lambda_{\infty}+2 \mu_{\infty}=1 \text { and } \mu_{\infty}>0 \\
I(a) \triangleq \frac{a}{1+a}, \quad k(a) \triangleq \frac{P^{\prime}(1+a)}{1+a}-1, \\
\widetilde{\mu}(a) \triangleq \mu(1+a)-\mu(1), \quad \widetilde{\lambda}(a) \triangleq \lambda(1+a)-\lambda(1) .
\end{gathered}
$$

Note that $I(a), k(a), \widetilde{\mu}(a)$ and $\widetilde{\lambda}(a)$ are smooth functions satisfying

$$
I(0)=k(0)=\widetilde{\mu}(0)=\widetilde{\lambda}(0)=0 .
$$

Now, we state our main result as follows.

Theorem 2.1. Let $d \geq 2$ and $p$ fulfill

$$
2 \leq p \leq \min \left(4, d^{*}\right) \quad \text { and, additionally, } p \neq 4 \text { if } d=2,
$$

where $d^{*} \triangleq 2 d /(d-2)$. Let $(\varrho, u)$ be the corresponding global solution of System (1.1) constructed in $[4,6,15,9]$ (or see Theorem 1.1 in [10]). Let the real number $\sigma_{1}$ satisfy

$$
1-\frac{d}{2}<\sigma_{1} \leq \sigma_{0} \quad \text { with } \sigma_{0} \triangleq \frac{2 d}{p}-\frac{d}{2} .
$$

There exists a positive constant $c=c(p, d, \lambda, \mu, P)$ such that if in addition the initial data $\left(a_{0} \triangleq \varrho_{0}-1, u_{0}\right)$ fulfill

$$
\mathcal{D}_{p, 0} \triangleq\left\|\left(a_{0}, u_{0}\right)\right\|_{\dot{B}_{2, \infty}^{-\sigma_{1}}}^{\ell} \leq c,
$$

then we have

$$
\mathcal{D}_{p}(t) \lesssim\left(\mathcal{D}_{p, 0}+\left\|\left(\nabla a_{0}, u_{0}\right)\right\|_{\dot{B}_{p, 1}^{\frac{d}{p}-1}}^{h}\right) \text { for all } t \geq 0
$$


where the functional $\mathcal{D}_{p}(t)$ is defined by

$$
\begin{aligned}
\mathcal{D}_{p}(t) \triangleq \sup _{\sigma \in\left[\varepsilon-\sigma_{1}, \frac{d}{2}+1\right]}\left\|\langle\tau\rangle^{\frac{\sigma_{1}+\sigma}{2}}(a, u)\right\|_{L_{t}^{\infty}\left(\dot{B}_{2,1}^{s}\right)}^{\ell} & \\
& +\left\|\langle\tau\rangle^{\alpha}(\nabla a, u)\right\|_{\widetilde{L}_{t}^{\infty}\left(\dot{B}_{p, 1}^{\frac{d}{p}-1}\right)}^{h}+\left\|\tau^{\alpha} \nabla u\right\|_{\widetilde{L}_{t}^{\infty}\left(\dot{B}_{p, 1}^{\frac{d}{p}}\right)}^{h}
\end{aligned}
$$

with $\alpha \triangleq \sigma_{1}+\frac{d}{2}+\frac{1}{2}-\varepsilon$ for sufficiently small $\varepsilon>0$.

Indeed, the analogue of (2.6) is not sharp in [10]. Our initial observation allows to gain more decay in time (for example, say $\tau^{-\beta}$ with $\beta \geq 1$ ) than $\tau^{-1}$ for the improved regularity of velocity. A natural question follows, since bounding the last two norms for high frequencies are absolutely interactional. Which values are optimal for the decay exponents $\alpha$ and $\beta$ respectively? To explore the optimality, we intend to develop the general low-frequency assumption for compressible fluid equations (1.1) and achieve (2.6) exhibiting optimal decay exponents for the high frequencies of solutions.

The statement of Theorem 2.1 deserves some comments.

(1) The assumption (2.3) enables us to enjoy relatively larger freedom on the choice of $\sigma_{1}$. For example, the value of $\sigma_{1}=\sigma_{0}=d / 2$ if taking $p=2$ is relevant in other contexts like the Boltzmann equation (see [35] by Sohinger and Strain), or hyperbolic systems with dissipation (see the joint works [39, 40] with Kawashima). We mention those previous works by Matsumura and Nishida [30], by Li and Zhang [20], by Guo and Wang [14], where different low-frequency assumptions were posed. Noticing that the Sobolev embeddings

$$
L^{1} \hookrightarrow \dot{B}_{1, \infty}^{0} \hookrightarrow \dot{B}_{2, \infty}^{-d / 2}, \quad \dot{H}^{-d / 2} \hookrightarrow \dot{B}_{2, \infty}^{-d / 2},
$$

we see that (2.4) is exactly less restrictive in contrast with that in [30, 20, 14].

(2) If replacing (2.4) by the slightly stronger assumption

$$
\left\|\left(a_{0}, u_{0}\right)\right\|_{\dot{B}_{2,1}^{-\sigma_{1}}}^{\ell} \leq c
$$

then we can take $\varepsilon=0$ : that is we can take $\alpha=\sigma_{1}+\frac{d}{2}+\frac{1}{2}$ and change the first term of $\mathcal{D}_{p}(t)$ by the slightly stronger norm

$$
\sup _{\sigma \in\left[-\sigma_{1}, \frac{d}{2}+1\right]}\left\|\langle\tau\rangle^{\frac{\sigma_{1}+\sigma}{2}}(a, u)\right\|_{\tilde{L}_{t}^{\infty}\left(\dot{B}_{2,1}^{\sigma}\right)}^{\ell} .
$$

We believe exponent $\alpha=\sigma_{1}+\frac{d}{2}+\frac{1}{2}$ as well as the upper bound $\sigma \leq d / 2+1$ for the first term of $\mathcal{D}_{p}$ to be optimal (see the very end of the present paper for more explanations). How to guess the lower bound of regularity $\sigma_{1}$ in the lowfrequency assumption? Actually, it may stem from the following elementary inequality:

$$
\int_{0}^{t}\langle t-\tau\rangle^{-\frac{\sigma_{1}+\sigma}{2}}\langle\tau\rangle^{-\delta} d \tau \lesssim\langle t\rangle^{-\frac{\sigma_{1}+\sigma}{2}}, \quad 0 \leq \frac{\sigma_{1}+\sigma}{2} \leq \delta, \quad \delta>1 .
$$

In our case, the minimum value of $\delta$ is $\sigma_{1} / 2+d / 4+1 / 2$, owing to $\sigma \leq d / 2+1$. Consequently, the desired lower bound of $\sigma_{1}$ just follows from the requirement $\sigma_{1} / 2+d / 4+1 / 2>1$ 
(3) In physical dimensions $d=2,3$, Condition (2.2) allows us to consider the case $p>d$, so that the regularity exponent $d / p-1$ for the velocity becomes negative. Our result thus applies to large highly oscillating initial velocities (see $[4,6]$ for more explanation), which is the essentially different ingredient in comparison with related decay works, see $[8,14,20,30,32]$ and references therein.

(4) Lastly, we would like to mention that (2.4) is of independent interest and thus has an application potential in mathematical analysis of classical Newtonian mechanics or complex fluids.

Let us present the strategy for proving Theorem 2.1. Since from [30], a usual approach getting the decay estimates of type (1.3) and (1.4) is to take advantage of $L^{1}-L^{2}$ decay estimates for the linearized system corresponding to the left-hand side of (2.1), treating the nonlinear right-hand side $(f, g)$ by means of Duhamel formula. In the critical regularity framework however, one cannot afford any loss of regularity for the high frequency part of the solution (and some terms like $u \cdot \nabla a$ induce a loss of one derivative as one cannot expect any smoothing for $a$, solution of a transport equation). The sketch of the proof of Theorem 2.1 is divided into three steps according to the three terms of $\mathcal{D}_{p}$. Due to $(2.4)$ in which $\sigma_{1}$ belongs to the whole range $\left(1-d / 2, \sigma_{0}\right]$, the low-frequency analysis (in the first step) is quite complicated. More concretely speaking, with aid of low and high frequency decompositions, we split the nonlinear term $(f, g)$ into $\left(f^{\ell}, g^{\ell}\right)$ and $\left(f^{h}, g^{h}\right)$ (see the context below). To handle $\left(f^{\ell}, g^{\ell}\right)$, some non classical product estimates in Besov spaces are well developed, see Lemma 4.2. Secondly, bounding the term $\left(f^{h}, g^{h}\right)$ seems to be more elaborate owing to the regularity of low frequencies. For clarity, Lemmas 4.3-4.4 are devoted to the non oscillation case $(2 \leq p \leq d)$, where we deal with cases $d / p-d / 2<\sigma_{1} \leq \sigma_{0}$ and $1-d / 2<\sigma_{1} \leq d / p-d / 2$ separately. Lemma 4.5 is only responsible for the oscillation case $(p>d)$. It is worth noting that those proofs of Lemmas 4.2-4.5 rely heavily on tricky product Besov estimates with respect to different Sobolev embeddings.

The purpose of the second and third steps is to track the optimal decay exponents for high frequencies with the general assumption (2.4). For convenience of reader, we follow from the fashion of [10] briefly and highlight new ingredients. Precisely, with the aid of the effective velocity (see for example, $[15,8]$ )

$$
w \triangleq \nabla(-\Delta)^{-1}(a-\operatorname{div} u)
$$

we get round the technical obstacle that there is a loss of one derivative of density in the second step. The last step is dedicated to establish gain of regularity and decay altogether for the high frequencies of the velocity, which strongly depends on the parabolic maximal regularity for the Lamé semi-group (that are the same as for the heat semi-group, see Remark 3.2).

By a by-product, Theorem 2.1 enables us to gain $L^{p}$ decay estimates of Ponce type (the slightly stronger $\dot{B}_{p, 1}^{0}$ norm in fact).

Corollary 2.1. The solution $(\varrho, u)$ constructed in Theorem 2.1 fulfills

$$
\begin{aligned}
\left\|\Lambda^{\sigma}(\varrho-1)\right\|_{L^{p}} & \lesssim\left(\mathcal{D}_{p, 0}+\left\|\left(\nabla a_{0}, u_{0}\right)\right\|_{\dot{B}_{p, 1}^{\frac{d}{p}-1}}^{h}\right)\langle t\rangle^{-\frac{\sigma_{1}+\sigma}{2}} \text { if }-\sigma_{1}<\sigma \leq \frac{d}{p}, \\
\left\|\Lambda^{\sigma} u\right\|_{L^{p}} & \lesssim\left(\mathcal{D}_{p, 0}+\left\|\left(\nabla a_{0}, u_{0}\right)\right\|_{\dot{B}_{p, 1}^{\frac{d}{p}-1}}^{h}\right)\langle t\rangle^{-\frac{\sigma_{1}+\sigma}{2}} \text { if }-\sigma_{1}<\sigma \leq \frac{d}{p}+1,
\end{aligned}
$$

where the pseudo differential operator $\Lambda^{s}$ is defined by $\Lambda^{s} f \triangleq \mathcal{F}^{-1}\left(|\xi|^{s} \mathcal{F} f\right)$. 
Furthermore, one can get the optimal decay estimates of $L^{q}-L^{r}$ type, as a consequence of improved Gagliardo-Nirenberg type inequalities which parallel the work of Sohinger and Strain [35] (see also [1], Chap. 2, or [40]).

Corollary 2.2. Let the assumptions of Theorem 2.1 be fulfilled with $p=2$. Then the corresponding solution $(\varrho, u)$ satisfies

$$
\left\|\Lambda^{l}(\varrho-1, u)\right\|_{L^{r}} \lesssim\left(\mathcal{D}_{2,0}+\left\|\left(\nabla a_{0}, u_{0}\right)\right\|_{\dot{B}_{2,1}^{\frac{d}{2}-1}}^{h}\right)\langle t\rangle^{-\frac{\sigma_{1}}{2}-\frac{d}{2}\left(\frac{1}{2}-\frac{1}{r}\right)-\frac{l}{2}}
$$

for $2 \leq r \leq \infty$ and $l \in \mathbb{R}$ satisfying $-\sigma_{1}<l+d\left(\frac{1}{2}-\frac{1}{r}\right) \leq \frac{d}{2}$.

Remark 2.1. The proofs of Corollaries 2.1-2.2 follow from essentially the argument as same as [10], and are thus omitted for brevity. The choice of derivative indices of velocity is somewhat relaxed thanks to the optimal exponents for high frequencies.

Finally, we end the section with an overview of our approach. Up to now, we present the optimal time-weighted estimates in $L^{p}$ critical spaces, for those constructed solutions (close to constant equilibrium) as in $[4,6,9,15]$. As a matter of fact, the vacuum mechanism is ruled out, so the low frequency linear analysis is dominate in our case. What happen, if the vacuum occurs (see e.g., [18]). The corresponding mathematical theory for viscous fluids is far away well known in critical spaces. In addition, providing an accurate large-time asymptotic description is still out of reach. For instance, one has to resort to detailed pointwise estimates on Green's function if characterizing the wave aspect of solutions. In this research line, we would like to mention the previous work by Zeng [41] devoted to the one-dimensional case. Hoff and Zumbrum [17] derived the $L^{p}$ decay rates towards diffusion waves in multi-dimensions, which are same as in (1.4). In [22], Liu and Wang exhibited pointwise estimates of diffusion waves with the optimal time-decay rate in odd dimension, that corresponds to the weak Huygens' principle.

The rest of the paper unfolds as follows: In Section 3, we briefly recall LittlewoodPaley decomposition, Besov spaces and related analysis tools. Section 4 is devoted to the proof of Theorem 2.1. In Section 5 (Appendix), we give some illustrations on the optimality of the regularity and decay exponents in the definition of $\mathcal{D}_{p}$.

\section{Preliminary}

Throughout the paper, $C>0$ stands for a harmless "constant". For brevity, we sometime write $u \lesssim v$ instead of $u \leq C v$. The notation $u \approx v$ means that $u \lesssim v$ and $v \lesssim u$. For any Banach space $X$ and $u, v \in X$, we agree that $\|(u, v)\|_{X} \triangleq\|u\|_{X}+\|v\|_{X}$. For all $T>0$ and $\rho \in[1,+\infty]$, we denote by $L_{T}^{\rho}(X) \triangleq L^{\rho}([0, T] ; X)$ the set of measurable functions $u:[0, T] \rightarrow X$ such that $t \mapsto\|u(t)\|_{X}$ is in $L^{\rho}(0, T)$.

To make the paper self-contained, let us recall Littlewood-Paley decomposition, Besov spaces and related analysis tools. More details may be found for example in Chap. 2 and Chap. 3 of [1]. Firstly, we introduce a homogeneous Littlewood-Paley decomposition. To do this, we fix some smooth radial non increasing function $\chi$ with Supp $\chi \subset B\left(0, \frac{4}{3}\right)$ and $\chi \equiv 1$ on $B\left(0, \frac{3}{4}\right)$, then set $\varphi(\xi)=\chi(\xi / 2)-\chi(\xi)$ so that

$$
\sum_{j \in \mathbb{Z}} \varphi\left(2^{-j} \cdot\right)=1 \text { in } \mathbb{R}^{d} \backslash\{0\} \text { and } \operatorname{Supp} \varphi \subset\left\{\xi \in \mathbb{R}^{d}: 3 / 4 \leq|\xi| \leq 8 / 3\right\} .
$$

Then homogeneous dyadic blocks $\dot{\Delta}_{j}$ are defined by

$$
\dot{\Delta}_{j} u \triangleq \varphi\left(2^{-j} D\right) u=\mathcal{F}^{-1}\left(\varphi\left(2^{-j} \cdot\right) \mathcal{F} u\right)=2^{j d} h\left(2^{j} \cdot\right) \star u \text { with } h \triangleq \mathcal{F}^{-1} \varphi .
$$


Therefore, one has the following homogeneous decomposition for any tempered distribution $u \in S^{\prime}\left(\mathbb{R}^{d}\right)$

$$
u=\sum_{j \in \mathbb{Z}} \dot{\Delta}_{j} u
$$

As it holds only modulo polynomials, it is convenient to consider the subspace of those tempered distributions $u$ such that

$$
\lim _{j \rightarrow-\infty}\left\|\dot{S}_{j} u\right\|_{L^{\infty}}=0
$$

where $\dot{S}_{j} f$ stands for the low frequency cut-off defined by $\dot{S}_{j} u \triangleq \chi\left(2^{-j} D\right) u$. Indeed, if equality (3.2) is fulfilled, then (3.1) holds in $S^{\prime}\left(\mathbb{R}^{d}\right)$. For convenience, we denote by $S_{0}^{\prime}\left(\mathbb{R}^{d}\right)$ the subspace of tempered distributions satisfying (3.2).

With the aid of the Littlewood-Paley decomposition, Besov spaces and related analysis tools will come into play in our context.

Definition 3.1. For $s \in \mathbb{R}$ and $1 \leq p, r \leq \infty$, the homogeneous Besov spaces $\dot{B}_{p, r}^{s}$ is defined by

where

$$
\dot{B}_{p, r}^{s} \triangleq\left\{u \in S_{0}^{\prime}:\|u\|_{\dot{B}_{p, r}^{s}}<+\infty\right\}
$$

$$
\|u\|_{\dot{B}_{p, r}^{s}} \triangleq\left\|\left(2^{j s}\left\|\dot{\Delta}_{j} u\right\|_{L^{p}}\right)\right\|_{\ell^{r}(\mathbb{Z})} .
$$

When studying the evolution PDEs, a class of mixed space-time Besov spaces are also used, which was first introduced by J.-Y. Chemin and N. Lerner [5] (see also [3] for the particular case of Sobolev spaces).

Definition 3.2. For $T>0, s \in \mathbb{R}, 1 \leq r, \theta \leq \infty$, the homogeneous Chemin-Lerner space $\widetilde{L}_{T}^{\theta}\left(\dot{B}_{p, r}^{s}\right)$ is defined by

where

$$
\widetilde{L}_{T}^{\theta}\left(\dot{B}_{p, r}^{s}\right) \triangleq\left\{u \in L^{\theta}\left(0, T ; S_{0}^{\prime}\right):\|u\|_{\widetilde{L}_{T}^{\theta}\left(\dot{B}_{p, r}^{s}\right)}<+\infty\right\},
$$

$$
\|u\|_{\widetilde{L}_{T}^{\theta}\left(\dot{B}_{p, r}^{s}\right)} \triangleq\left\|\left(2^{j s}\left\|\dot{\Delta}_{j} u\right\|_{L_{T}^{\theta}\left(L^{p}\right)}\right)\right\|_{\ell^{r}(\mathbb{Z})} .
$$

For notational simplicity, index $T$ is omitted if $T=+\infty$. We also use the following functional space:

$$
\widetilde{\mathcal{C}}_{b}\left(\mathbb{R}_{+} ; \dot{B}_{p, r}^{s}\right) \triangleq\left\{u \in \mathcal{C}\left(\mathbb{R}_{+} ; \dot{B}_{p, r}^{s}\right) \text { s.t }\|u\|_{\widetilde{L}^{\infty}\left(\dot{B}_{p, r}^{s}\right)}<+\infty\right\} .
$$

The above norm (3.4) may be compared with those of the standard spaces $L_{T}^{\theta}\left(\dot{B}_{p, r}^{s}\right)$ by means of Minkowski's inequality.

Remark 3.1. It holds that

$$
\|u\|_{\widetilde{L}_{T}^{\theta}\left(B_{p, r}^{s}\right)} \leq\|u\|_{L_{T}^{\theta}\left(B_{p, r}^{s}\right)} \quad \text { if } r \geq \theta ; \quad\|u\|_{\widetilde{L}_{T}^{\theta}\left(B_{p, r}^{s}\right)} \geq\|u\|_{L_{T}^{\theta}\left(B_{p, r}^{s}\right)} \quad \text { if } r \leq \theta .
$$

Restricting the above norms (3.3) and (3.4) to the low or high frequencies parts of distributions will be fundamental in our approach. For example, let us fix some integer $j_{0}$ (the value of which will follow from the proof of the main theorem) and put ${ }^{1}$

$$
\|u\|_{\dot{B}_{p, 1}^{s}}^{\ell} \triangleq \sum_{j \leq j_{0}} 2^{j s}\left\|\dot{\Delta}_{k} f\right\|_{L^{p}} \text { and }\|u\|_{\dot{B}_{p, 1}^{s}}^{h} \triangleq \sum_{j \geq j_{0}-1} 2^{j s}\left\|\dot{\Delta}_{j} u\right\|_{L^{p}}
$$

\footnotetext{
${ }^{1}$ Note that for technical reasons, we need a small overlap between low and high frequencies.
} 


$$
\|u\|_{\widetilde{L}_{T}^{\infty}\left(\dot{B}_{p, 1}^{s}\right)}^{\ell} \triangleq \sum_{j \leq j_{0}} 2^{j s}\left\|\dot{\Delta}_{j} u\right\|_{L_{T}^{\infty}\left(L^{p}\right)} \text { and }\|u\|_{\widetilde{L}_{T}^{\infty}\left(\dot{B}_{p, 1}^{s}\right)}^{h} \triangleq \sum_{j \geq j_{0}-1} 2^{j s}\left\|\dot{\Delta}_{j} u\right\|_{L_{T}^{\infty}\left(L^{p}\right)} .
$$

We often use the following classical properties (see [1]):

- Scaling invariance: For any $s \in \mathbb{R}$ and $(p, r) \in[1, \infty]^{2}$, there exists a constant $C=C(s, p, r, d)$ such that for all $\lambda>0$ and $u \in \dot{B}_{p, r}^{s}$, we have

$$
C^{-1} \lambda^{s-\frac{d}{p}}\|u\|_{\dot{B}_{p, r}^{s}} \leq\|u(\lambda \cdot)\|_{\dot{B}_{p, r}^{s}} \leq C \lambda^{s-\frac{d}{p}}\|u\|_{\dot{B}_{p, r}^{s}} .
$$

- Completeness: $\dot{B}_{p, r}^{s}$ is a Banach space whenever $s<\frac{d}{p}$ or $s \leq \frac{d}{p}$ and $r=1$.

- Interpolation: The following inequality is satisfied for $1 \leq p, r_{1}, r_{2}, r \leq \infty, s_{1} \neq s_{2}$ and $\theta \in(0,1)$ :

with $\frac{1}{r}=\frac{\theta}{r_{2}}+\frac{1-\theta}{r_{2}}$.

$$
\|u\|_{\dot{B}_{p, r}^{\theta s_{1}+(1-\theta) s_{2}}} \lesssim\|u\|_{\dot{B}_{p, r_{1}}^{s_{1}}}^{\theta}\|u\|_{\dot{B}_{p, r_{2}}^{s_{2}}}^{1-\theta}
$$

- Action of Fourier multipliers: If $F$ is a smooth homogeneous of degree $m$ function on $\mathbb{R}^{d} \backslash\{0\}$ then

$$
F(D): \dot{B}_{p, r}^{s} \rightarrow \dot{B}_{p, r}^{s-m}
$$

Proposition 3.1. (Embedding for Besov spaces on $\mathbb{R}^{d}$ )

- For any $p \in[1, \infty]$ we have the continuous embedding $\dot{B}_{p, 1}^{0} \hookrightarrow L^{p} \hookrightarrow \dot{B}_{p, \infty}^{0}$.

- If $\sigma \in \mathbb{R}, 1 \leq p_{1} \leq p_{2} \leq \infty$ and $1 \leq r_{1} \leq r_{2} \leq \infty$, then $\dot{B}_{p_{1}, r_{1}}^{\sigma} \hookrightarrow \dot{B}_{p_{2}, r_{2}}^{\sigma-d\left(\frac{1}{p_{1}}-\frac{1}{p_{2}}\right)}$.

- The space $\dot{B}_{p, 1}^{\frac{d}{p}}$ is continuously embedded in the set of bounded continuous functions (going to zero at infinity if, additionally, $p<\infty$ ).

The following product estimates in Besov spaces play a fundamental role in our analysis of the bilinear terms of (2.1).

Proposition 3.2. ([1, 10]) Let $s>0$ and $1 \leq p, r \leq \infty$. Then $\dot{B}_{p, r}^{s} \cap L^{\infty}$ is an algebra and

$$
\|u v\|_{\dot{B}_{p, r}^{s}} \lesssim\|u\|_{L^{\infty}}\|v\|_{\dot{B}_{p, r}^{s}}+\|v\|_{L^{\infty}}\|u\|_{\dot{B}_{p, r}^{s}} .
$$

Let the real numbers $s_{1}, s_{2}, p_{1}$ and $p_{2}$ be such that

$$
s_{1}+s_{2}>0, \quad s_{1} \leq \frac{d}{p_{1}}, \quad s_{2} \leq \frac{d}{p_{2}}, \quad s_{1} \geq s_{2}, \quad \frac{1}{p_{1}}+\frac{1}{p_{2}} \leq 1 .
$$

Then we have

$$
\|u v\|_{\dot{B}_{q, 1}^{s_{2}}} \lesssim\|u\|_{\dot{B}_{p_{1}, 1}^{s_{1}}}\|v\|_{\dot{B}_{p_{2}, 1}^{s_{2}}} \quad \text { with } \quad \frac{1}{q}=\frac{1}{p_{1}}+\frac{1}{p_{2}}-\frac{s_{1}}{d} .
$$

Additionally, for exponents $s>0$ and $1 \leq p_{1}, p_{2}, q \leq \infty$ satisfying

$$
\frac{d}{p_{1}}+\frac{d}{p_{2}}-d \leq s \leq \min \left(\frac{d}{p_{1}}, \frac{d}{p_{2}}\right) \quad \text { and } \quad \frac{1}{q}=\frac{1}{p_{1}}+\frac{1}{p_{2}}-\frac{s}{d},
$$

we have

$$
\|u v\|_{\dot{B}_{q, \infty}^{-s}} \lesssim\|u\|_{\dot{B}_{p_{1}, 1}^{s}}\|v\|_{\dot{B}_{p_{2}, \infty}^{-s}} .
$$

Proposition 3.2 are not enough to bound the possible case $p>d$ in the proof of Theorem 2.1, so we have the following non-classical product estimates. 
Proposition 3.3. ([10]) Let $j_{0} \in \mathbb{Z}$, and denote $z^{\ell} \triangleq \dot{S}_{j_{0}} z, z^{h} \triangleq z-z^{\ell}$ and, for any $s \in \mathbb{R}$,

$$
\|z\|_{\dot{B}_{2, \infty}^{s}}^{\ell} \triangleq \sup _{j \leq j_{0}} 2^{j s}\left\|\dot{\Delta}_{j} z\right\|_{L^{2}}
$$

There exists a universal integer $N_{0}$ such that for any $2 \leq p \leq 4$ and $s>0$, we have

$$
\begin{aligned}
\left\|u v^{h}\right\|_{\dot{B}_{2, \infty}^{-\sigma_{0}}}^{\ell} & \leq C\left(\|u\|_{\dot{B}_{p, 1}^{s}}+\left\|\dot{S}_{j_{0}+N_{0}} u\right\|_{L^{p^{*}}}\right)\left\|v^{h}\right\|_{\dot{B}_{p, \infty}^{-s}} \\
\left\|u^{h} v\right\|_{\dot{B}_{2, \infty}^{-\sigma_{0}}}^{\ell} & \leq C\left(\left\|u^{h}\right\|_{\dot{B}_{p, 1}^{s}}+\left\|\dot{S}_{j_{0}+N_{0}} u^{h}\right\|_{L^{p^{*}}}\right)\|v\|_{\dot{B}_{p, \infty}^{-s}}
\end{aligned}
$$

with $\sigma_{0} \triangleq \frac{2 d}{p}-\frac{d}{2}$ and $\frac{1}{p^{*}} \triangleq \frac{1}{2}-\frac{1}{p}$, and $C$ depending only on $j_{0}, d$ and $s$.

System (2.1) also involves compositions of functions (through $I(a), k(a), \widetilde{\lambda}(a)$ and $\widetilde{\mu}(a))$ and they are bounded according to the following conclusion.

Proposition 3.4. Let $F: \mathbb{R} \rightarrow \mathbb{R}$ be smooth with $F(0)=0$. For all $1 \leq p, r \leq \infty$ and $s>0$ we have $F(u) \in \dot{B}_{p, r}^{s} \cap L^{\infty}$ for $u \in \dot{B}_{p, r}^{s} \cap L^{\infty}$, and

$$
\|F(u)\|_{\dot{B}_{p, r}^{s}} \leq C\|u\|_{\dot{B}_{p, r}^{s}}
$$

with $C$ depending only on $\|u\|_{L^{\infty}}, F^{\prime}$ (and higher derivatives), $s, p$ and $d$.

In the case $s>-\min \left(\frac{d}{p}, \frac{d}{p^{\prime}}\right)$ then $u \in \dot{B}_{p, r}^{s} \cap \dot{B}_{p, 1}^{\frac{d}{p}}$ implies that $F(u) \in \dot{B}_{p, r}^{s} \cap \dot{B}_{p, 1}^{\frac{d}{p}}$, and we have

$$
\|F(u)\|_{\dot{B}_{p, r}^{s}} \leq C\left(1+\|u\|_{\dot{B}_{p, 1}^{\frac{d}{p}}}\right)\|u\|_{\dot{B}_{p, r}^{s}} .
$$

Let us now recall the following classical Bernstein inequality:

$$
\left\|D^{k} u\right\|_{L^{b}} \leq C^{1+k} \lambda^{k+d\left(\frac{1}{a}-\frac{1}{b}\right)}\|u\|_{L^{a}}
$$

that holds for all function $u$ such that $\operatorname{Supp} \mathcal{F} u \subset\left\{\xi \in \mathbb{R}^{d}:|\xi| \leq R \lambda\right\}$ for some $R>0$ and $\lambda>0$, if $k \in \mathbb{N}$ and $1 \leq a \leq b \leq \infty$.

More generally, if we assume $u$ to satisfy $\operatorname{Supp} \mathcal{F} u \subset\left\{\xi \in \mathbb{R}^{d}: R_{1} \lambda \leq|\xi| \leq R_{2} \lambda\right\}$ for some $0<R_{1}<R_{2}$ and $\lambda>0$, then for any smooth homogeneous of degree $m$ function $A$ on $\mathbb{R}^{d} \backslash\{0\}$ and $1 \leq a \leq \infty$, we have (see e.g. Lemma 2.2 in [1]):

$$
\|A(D) u\|_{L^{a}} \lesssim \lambda^{m}\|u\|_{L^{a}} .
$$

An obvious consequence of (3.7) and (3.8) is that $\left\|D^{k} u\right\|_{\dot{B}_{p, r}^{s}} \approx\|u\|_{\dot{B}_{p, r}^{s+k}}$ for all $k \in \mathbb{N}$.

A time-dependent version of the following commutator estimate has been used in the second step of the proof of Theorem 2.1.

Proposition 3.5. ([10]) Let $1 \leq p, p_{1} \leq \infty$ and

$$
-\min \left(\frac{d}{p_{1}}, \frac{d}{p^{\prime}}\right)<s \leq 1+\min \left(\frac{d}{p}, \frac{d}{p_{1}}\right) \quad \text { with } \quad \frac{1}{p}+\frac{1}{p^{\prime}}=1 .
$$

There exists a constant $C>0$ depending only on s such that for all $j \in \mathbb{Z}$ and $\ell \in$ $\{1, \cdots, d\}$, we have

$$
\left\|\left[v \cdot \nabla, \partial_{\ell} \dot{\Delta}_{j}\right] a\right\|_{L^{p}} \leq C c_{j} 2^{-j(s-1)}\|\nabla v\|_{\dot{B}_{p_{1}, 1}^{\frac{d}{p_{1}}}}\|\nabla a\|_{\dot{B}_{p, 1}^{s-1}},
$$

where the commutator $[\cdot, \cdot]$ is defined by $[f, g]=f g-g f$ and $\left(c_{j}\right)_{j \in \mathbb{Z}}$ denotes a sequence such that $\left\|\left(c_{j}\right)\right\|_{\ell^{1}} \leq 1$. 
Let us finally recall the following parabolic regularity estimate for the heat equation to end this section.

Proposition 3.6. Let $s \in \mathbb{R},(p, r) \in[1, \infty]^{2}$ and $1 \leq \rho_{2} \leq \rho_{1} \leq \infty$. Let u satisfy

$$
\left\{\begin{array}{l}
\partial_{t} u-\mu \Delta u=f \\
u_{\mid t=0}=u_{0}
\end{array}\right.
$$

Then for all $T>0$ the following a priori estimate is fulfilled:

$$
\mu^{\frac{1}{\rho_{1}}}\|u\|_{\widetilde{L}_{T}^{\rho_{1}}\left(\dot{B}_{p, r}^{\left.s+\frac{2}{\rho_{1}}\right)}\right.} \lesssim\left\|u_{0}\right\|_{\dot{B}_{p, r}^{s}}+\mu^{\frac{1}{\rho_{2}}-1}\|f\|_{\widetilde{L}_{T}^{\rho_{2}}\left(\dot{B}_{p, r}^{s-2+\frac{2}{\rho_{2}}}\right)} .
$$

Remark 3.2. The solutions to the following Lamé system

$$
\left\{\begin{array}{l}
\partial_{t} u-\mu \Delta u-(\lambda+\mu) \nabla \operatorname{div} u=f \\
u_{\mid t=0}=u_{0}
\end{array}\right.
$$

where $\lambda$ and $\mu$ are constant coefficients such that $\mu>0$ and $\lambda+2 \mu>0$, also fulfill (3.9) (up to the dependence w.r.t. the viscosity). Indeed, if we denote by $\mathcal{P} \triangleq$ $\mathrm{Id}+\nabla(-\Delta)^{-1} \operatorname{div}$ and $\mathcal{Q} \triangleq \mathrm{Id}-\mathcal{P}$ the orthogonal projectors over divergence-free and potential vector fields, then we see both $\mathcal{P} u$ and $\mathcal{Q} u$ satisfy the heat equation, as it can easily be observed by applying $\mathcal{P}$ and $\mathcal{Q}$ to (3.10).

\section{The PROOF OF TIME-DECAY ESTIMATES}

This section is devoted to the proof of Theorem 2.1 taking for granted the global-intime existence result of Theorem 1.1 in [10]. We denote the energy norm as follows:

$$
\begin{aligned}
\mathcal{E}_{p}(t) \triangleq & \|(a, u)\|_{\widetilde{L}_{t}^{\infty}\left(\dot{B}_{2,1}^{\frac{d}{2}-1}\right)}^{\ell}+\|(a, u)\|_{L_{t}^{1}\left(\dot{B}_{2,1}^{\frac{d}{2}+1}\right)}^{\ell}+\|(\nabla a, u)\|_{\widetilde{L}_{t}^{\infty}\left(\dot{B}_{p, 1}^{\frac{d}{p}-1}\right)}^{h} \\
& +\|(a, \nabla u)\|_{L_{t}^{1}\left(\dot{B}_{p, 1}^{\frac{d}{p}}\right)}^{h} .
\end{aligned}
$$

In what follows, we shall use repeatedly that for $0 \leq s_{1} \leq s_{2}$ with $s_{2}>1$, we have

$$
\int_{0}^{t}\langle t-\tau\rangle^{-s_{1}} \tau^{-\theta}\langle\tau\rangle^{\theta-s_{2}} d \tau \lesssim\langle t\rangle^{-s_{1}} \text { if } 0 \leq \theta<1 .
$$

Let us keep in mind that the global solution $(a, u)$ given by Theorem 2.1 satisfies

$$
\|a\|_{\widetilde{L}_{t}^{\infty}\left(\dot{B}_{p, 1}^{\frac{d}{p}}\right)} \leq c \ll 1 \text { for all } t \geq 0 .
$$

4.1. First step: Bounds for the low frequencies. Let $(\mathcal{G}(t))_{t \geq 0}$ be the semi-group associated with the left-hand side of (2.1). The standard Duhamel principle gives

$$
\left(\begin{array}{l}
a(t) \\
u(t)
\end{array}\right)=\mathcal{G}(t)\left(\begin{array}{l}
a_{0} \\
u_{0}
\end{array}\right)+\int_{0}^{t} \mathcal{G}(t-\tau)\left(\begin{array}{c}
f(\tau) \\
g(\tau)
\end{array}\right) d \tau
$$

First of all, we state smoothing estimate of the linearized solution $\left(a_{L}, u_{L}\right) \triangleq \mathcal{G}(t)\left(a_{0}, u_{0}\right)$, which behaves like that of heat kernel.

Lemma 4.1. Let $\left(a_{L}, u_{L}\right)$ be the solution to the linear system

$$
\left\{\begin{array}{l}
\partial_{t} a_{L}+\operatorname{div} u_{L}=0 \\
\partial_{t} u_{L}-\mathcal{A} u_{L}+\nabla a_{L}=0
\end{array}\right.
$$

with the initial data

$$
\left.\left(a_{L}, u_{L}\right)\right|_{t=0}=\left(a_{0}, u_{0}\right) .
$$


Then, for any $j_{0} \in \mathbb{Z}$, there exists a positive constant $c_{0}=c_{0}\left(\lambda_{\infty}, \mu_{\infty}, j_{0}\right)$ such that

$$
\left\|\left(a_{L, j}, u_{L, j}\right)(t)\right\|_{L^{2}} \lesssim e^{-c_{0} 2^{2 j} t}\left\|\left(a_{0, j}, u_{0, j}\right)\right\|_{L^{2}}
$$

for $t \geq 0$ and $j \leq j_{0}$, where we set $z_{j}=\dot{\Delta}_{j} z$ for any $z \in S^{\prime}\left(\mathbb{R}^{d}\right)$.

The reader is referred to [4] for the proof of Lemma 4.1. Set $U \triangleq(a, u)$ and $U_{0} \triangleq$ $\left(a_{0}, u_{0}\right)$. It is not difficult to deduce that for $\sigma_{1}+\sigma>0$ (see [10])

$$
\sup _{t \geq 0} t^{\frac{\sigma_{1}+\sigma}{2}}\left\|\mathcal{G}(t) U_{0}\right\|_{\dot{B}_{2,1}^{\sigma}}^{\ell} \lesssim\left\|U_{0}\right\|_{\dot{B}_{2, \infty}^{-\sigma_{1}}}^{\ell} .
$$

Moreover, it is clear that for $\sigma+\sigma_{1}>0$,

$$
\left\|\mathcal{G}(t) U_{0}\right\|_{\dot{B}_{2,1}^{\sigma}}^{\ell} \lesssim\left\|U_{0}\right\|_{\dot{B}_{2, \infty}^{-\sigma_{1}}}^{\ell} \sum_{j \leq j_{0}} 2^{j\left(\sigma_{1}+\sigma\right)} \lesssim\left\|U_{0}\right\|_{\dot{B}_{2, \infty}^{-\sigma_{1}}}^{\ell} .
$$

Hence, setting $\langle t\rangle \triangleq \sqrt{1+t^{2}}$, we arrive at

$$
\sup _{t \geq 0}\langle t\rangle^{\frac{\sigma_{1}+\sigma}{2}}\left\|\mathcal{G}(t) U_{0}\right\|_{\dot{B}_{2,1}^{\sigma}}^{\ell} \lesssim\left\|U_{0}\right\|_{\dot{B}_{2, \infty}^{-\sigma_{1}}}^{\ell}
$$

Furthermore, we get from Duhamel's formula that

$$
\left\|\int_{0}^{t} \mathcal{G}(t-\tau)(f, g)(\tau) d \tau\right\|_{\dot{B}_{2,1}^{\sigma}}^{\ell} \lesssim \int_{0}^{t}\langle t-\tau\rangle^{-\frac{\sigma_{1}+\sigma}{2}}\|(f, g)(\tau)\|_{\dot{B}_{2, \infty}^{-\sigma_{1}}}^{\ell} d \tau .
$$

To bound the above time-weighted integral on the right side, we have the following proposition.

Proposition 4.1. If $p$ satisfies (2.2), then it holds that for all $t \geq 0$,

$$
\int_{0}^{t}\langle t-\tau\rangle^{-\frac{\sigma_{1}+\sigma}{2}}\|(f, g)(\tau)\|_{\dot{B}_{2, \infty}^{-\sigma_{1}}}^{\ell} d \tau \lesssim\langle t\rangle^{-\frac{\sigma_{1}+\sigma}{2}}\left(\mathcal{D}_{p}^{2}(t)+\mathcal{E}_{p}^{2}(t)\right),
$$

provided that $-\sigma_{1}<\sigma \leq \frac{d}{2}+1$, where $\mathcal{E}_{p}(t)$ and $\mathcal{D}_{p}(t)$ have been defined by (4.1) and (2.6), respectively.

For clarity, we divide the proof of Proposition 4.1 into several lemmas. It is convenient to decompose $f$ and $g$ in terms of low-frequency and high-frequency as follows:

$$
f=f^{\ell}+f^{h}
$$

with

$$
f^{\ell} \triangleq-a \operatorname{div} u^{\ell}-u \cdot \nabla a^{\ell}, \quad f^{h} \triangleq-a \operatorname{div} u^{h}-u \cdot \nabla a^{h}
$$

and

$$
g=g^{\ell}+g^{h}
$$

with

$$
\begin{gathered}
g^{\ell} \triangleq-u \cdot \nabla u^{\ell}-k(a) \nabla a^{\ell}+g_{3}\left(a, u^{\ell}\right)+g_{4}\left(a, u^{\ell}\right), \\
g^{h} \triangleq-u \cdot \nabla u^{h}-k(a) \nabla a^{h}+g_{3}\left(a, u^{h}\right)+g_{4}\left(a, u^{h}\right),
\end{gathered}
$$

where

$$
\begin{aligned}
& g_{3}(a, v)=\frac{1}{1+a}(2 \widetilde{\mu}(a) \operatorname{div} D(v)+\widetilde{\lambda}(a) \nabla \operatorname{div} v)-I(a) \mathcal{A} v, \\
& g_{4}(a, v)=\frac{1}{1+a}\left(2 \widetilde{\mu}^{\prime}(a) D(v) \cdot \nabla a+\widetilde{\lambda}^{\prime}(a) \operatorname{div} v \nabla a\right)
\end{aligned}
$$


and

$$
z^{\ell} \triangleq \sum_{j<j_{0}} \dot{\Delta}_{j} z, \quad z^{h} \triangleq z-z^{\ell} \text { for } z=a, u .
$$

Lemma 4.2. If $p$ satisfies (2.2), then it holds that for all $t \geq 0$,

$$
\int_{0}^{t}\langle t-\tau\rangle^{-\frac{\sigma_{1}+\sigma}{2}}\left\|\left(f^{\ell}, g^{\ell}\right)(\tau)\right\|_{\dot{B}_{2, \infty}^{-\sigma_{1}}}^{\ell} d \tau \lesssim\langle t\rangle^{-\frac{\sigma_{1}+\sigma}{2}}\left(\mathcal{D}_{p}^{2}(t)+\mathcal{E}_{p}^{2}(t)\right) .
$$

Proof. To bound the six terms of $f$ and $g$ with $a^{\ell}$ or $u^{\ell}$, we first claim the following two non classical product estimates:

$$
\begin{aligned}
& \|F G\|_{\dot{B}_{2, \infty}^{-\sigma_{1}}} \lesssim\|F\|_{\dot{B}_{p, 1}^{\frac{d}{p}}}\|G\|_{\dot{B}_{2,1}^{-\sigma_{1}}}, \\
& \|F G\|_{\dot{B}_{2, \infty}^{\frac{d}{p}-\frac{d}{2}-\sigma_{1}}} \lesssim\|F\|_{\dot{B}_{p, 1}^{\frac{d}{p}-\frac{d}{2}-\sigma_{1}}}\|G\|_{\dot{B}_{2,1}^{\frac{d}{p}}} .
\end{aligned}
$$

Indeed, if $\sigma_{1}=\sigma_{0}$, then (4.9) stems from the last term of Proposition 3.2 with $s=\frac{d}{p}, q=p_{1}=2, p_{2}=p$. If $1-d / 2<\sigma_{1}<\sigma_{0}$, then (4.9) follows from the second term of Proposition 3.2 with

$$
\frac{d}{p} \triangleq s_{1} \geq s_{2} \triangleq \frac{d}{p}-\frac{d}{2}-\sigma_{1}, \quad q=p_{1}=2 \text { and } p_{2}=p
$$

satisfying $s_{1}+s_{2}>0$. As for (4.8), if $p=2$ then it coincides with (4.9). If $2<p \leq d^{*}$ (see (2.2)), then (4.8) is followed by the second item of Proposition 3.2 with

$$
\frac{d}{p} \triangleq s_{1} \geq s_{2} \triangleq-\sigma_{1}, \quad q=p_{2}=2 \text { and } p_{1}=p
$$

satisfying $s_{1}+s_{2}>0$.

In addition, due to $\alpha \triangleq \sigma_{1}+\frac{d}{2}+\frac{1}{2}-\varepsilon \geq \frac{\sigma_{1}}{2}+\frac{d}{4}$ for small enough $\varepsilon>0$, the embedding in Proposition 3.1 and the definition of $\mathcal{D}_{p}(t)$, we obtain

$$
\begin{aligned}
\left\|\left(a, u^{\ell}\right)(\tau)\right\|_{\dot{B}_{p, 1}^{\frac{d}{p}}} & \lesssim\langle\tau\rangle^{-\frac{\sigma_{1}}{2}-\frac{d}{4}}\left(\langle\tau\rangle^{\frac{\sigma_{1}}{2}+\frac{d}{4}}\|(a, u)(\tau)\|_{\dot{B}_{2,1}^{\frac{d}{2}}}^{\ell}+\langle\tau\rangle^{\alpha}\|a(\tau)\|_{\dot{B}_{p, 1}^{\frac{d}{p}}}^{h}\right) \\
& \lesssim\langle\tau\rangle^{-\frac{\sigma_{1}}{2}-\frac{d}{4}} \mathcal{D}_{p}(\tau)
\end{aligned}
$$

and also that, owing to $-\sigma_{1}<1-\sigma_{1}<2-\sigma_{1}<\frac{d}{2}+1$,

$$
\left\|\nabla\left(a^{\ell}, u^{\ell}\right)(\tau)\right\|_{\dot{B}_{2,1}^{-\sigma_{1}}} \lesssim\langle\tau\rangle^{-\frac{1}{2}} \mathcal{D}_{p}(\tau), \quad\left\|\nabla^{2} u^{\ell}(\tau)\right\|_{\dot{B}_{2,1}^{-\sigma_{1}}} \lesssim\langle\tau\rangle^{-1} \mathcal{D}_{p}(\tau) .
$$

Observe that $\frac{d}{p}-\frac{d}{2}-\sigma_{1}<\frac{d}{p}-1$ and $\alpha>1$ for small enough $\varepsilon>0$, we have by embedding,

$$
\left\|\left(\nabla a, u^{h}\right)(\tau)\right\|_{\dot{B}_{p, 1}^{\frac{d}{p}-\frac{d}{2}-\sigma_{1}}} \lesssim\left\|\nabla a^{\ell}(\tau)\right\|_{\dot{B}_{2,1}^{-\sigma_{1}}}+\left\|\left(\nabla a^{h}, u^{h}\right)(\tau)\right\|_{\dot{B}_{p, 1}^{\frac{d}{p}-1}} \lesssim\langle\tau\rangle^{-\frac{1}{2}} \mathcal{D}_{p}(\tau)
$$

and, thanks to $-\sigma_{1}<\frac{d}{2}-1<\frac{d}{2} \leq \frac{d}{p}+1 \leq \frac{d}{2}+1$,

$$
\left\|\nabla\left(a^{\ell}, u^{\ell}\right)(\tau)\right\|_{\dot{B}_{2,1}^{\frac{d}{p}}} \lesssim\langle\tau\rangle^{-\frac{\sigma_{1}}{2}-\frac{d}{2 p}-\frac{1}{2}} \mathcal{D}_{p}(\tau) .
$$


Now, we begin with prove (4.7). To bound the term with $a \operatorname{div} u^{\ell}$, we write that, owing to $(4.8),(4.10)$ and $(4.11)$,

$$
\begin{aligned}
\int_{0}^{t}\langle t-\tau\rangle^{-\frac{\sigma_{1}+\sigma}{2}}\left\|a \operatorname{div} u^{\ell}\right\|_{\dot{B}_{2, \infty}^{-\sigma_{1}}}^{\ell} d \tau & \lesssim \int_{0}^{t}\langle t-\tau\rangle^{-\frac{\sigma_{1}+\sigma}{2}}\|a\|_{\dot{B}_{p, 1}^{\frac{d}{p}}}\left\|\operatorname{div} u^{\ell}\right\|_{\dot{B}_{2,1}^{-\sigma_{1}}} d \tau \\
& \lesssim \mathcal{D}_{p}^{2}(t) \int_{0}^{t}\langle t-\tau\rangle^{-\frac{\sigma_{1}+\sigma}{2}}\langle\tau\rangle^{-\frac{\sigma_{1}}{2}-\frac{d}{4}-\frac{1}{2}} d \tau .
\end{aligned}
$$

As $\frac{\sigma_{1}}{2}+\frac{d}{4}+\frac{1}{2}>1$ and $\frac{\sigma_{1}}{2}+\frac{d}{4}+\frac{1}{2} \geq \frac{\sigma_{1}+\sigma}{2}$ for $\sigma_{1}$ satisfying (2.3) and $\sigma \leq \frac{d}{2}+1$, inequality (4.2) implies that

$$
\int_{0}^{t}\langle t-\tau\rangle^{-\frac{\sigma_{1}+\sigma}{2}}\left\|a \operatorname{div} u^{\ell}\right\|_{\dot{B}_{2, \infty}^{-\sigma_{1}}}^{\ell} d \tau \lesssim\langle t\rangle^{-\frac{\sigma_{1}+\sigma}{2}} \mathcal{D}_{p}^{2}(t) .
$$

Regarding the term with $k(a) \nabla a^{\ell}$, with the aid of Proposition 3.4, (4.2), (4.8) and (4.10)-(4.11), we similarly get

$$
\begin{aligned}
\int_{0}^{t}\langle t-\tau\rangle^{-\frac{\sigma_{1}+\sigma}{2}}\left\|k(a) \nabla a^{\ell}\right\|_{\dot{B}_{2, \infty}^{-\sigma_{1}}}^{\ell} d \tau & \lesssim \int_{0}^{t}\langle t-\tau\rangle^{-\frac{\sigma_{1}+\sigma}{2}}\|a\|_{\dot{B}_{p, 1}^{\frac{d}{p}}}\left\|\nabla a^{\ell}\right\|_{\dot{B}_{2,1}^{-\sigma_{1}}} d \tau \\
& \lesssim \mathcal{D}_{p}^{2}(t) \int_{0}^{t}\langle t-\tau\rangle^{-\frac{\sigma_{1}+\sigma}{2}}\langle\tau\rangle^{-\frac{\sigma_{1}}{2}-\frac{d}{4}-\frac{1}{2}} d \tau \\
& \lesssim\langle t\rangle^{-\frac{\sigma_{1}+\sigma}{2}} \mathcal{D}_{p}^{2}(t) .
\end{aligned}
$$

Regarding the term with $g_{3}\left(a, u^{\ell}\right)$, it follows from Proposition 3.4, (4.2), (4.8) and (4.10)-(4.11) that

$$
\begin{aligned}
\int_{0}^{t}\langle t-\tau\rangle^{-\frac{\sigma_{1}+\sigma}{2}}\left\|g_{3}\left(a, u^{\ell}\right)\right\|_{\dot{B}_{2, \infty}^{-\sigma_{1}}}^{\ell} d \tau & \lesssim \int_{0}^{t}\langle t-\tau\rangle^{-\frac{\sigma_{1}+\sigma}{2}}\|a\|_{\dot{B}_{p, 1}^{\frac{d}{p}}}\left\|\nabla^{2} u^{\ell}\right\|_{\dot{B}_{2,1}^{-\sigma_{1}}} d \tau \\
& \lesssim \mathcal{D}_{p}^{2}(t) \int_{0}^{t}\langle t-\tau\rangle^{-\frac{\sigma_{1}+\sigma}{2}}\langle\tau\rangle^{-\frac{\sigma_{1}}{2}-\frac{d}{4}-1} d \tau \\
& \lesssim\langle t\rangle^{-\frac{\sigma_{1}+\sigma}{2}} \mathcal{D}_{p}^{2}(t) .
\end{aligned}
$$

We decompose $u \cdot \nabla a^{\ell}=u^{\ell} \cdot \nabla a^{\ell}+u^{h} \cdot \nabla a^{\ell}$ and $u \cdot \nabla u^{\ell}=u^{\ell} \cdot \nabla u^{\ell}+u^{h} \cdot \nabla u^{\ell}$. Bounding $u^{\ell} \cdot \nabla a^{\ell}$ and $u^{\ell} \cdot \nabla u^{\ell}$ essentially follow from the same procedure as $a \operatorname{div} u^{\ell}$, we omit them. For the term containing $u^{h} \cdot \nabla a^{\ell}$, we note that, owing to (4.9),

$$
\int_{0}^{t}\langle t-\tau\rangle^{-\frac{\sigma_{1}+\sigma}{2}}\left\|u^{h} \cdot \nabla a^{\ell}\right\|_{\dot{B}_{2, \infty}^{\frac{d}{p}-\frac{d}{2}-\sigma_{1}}}^{\ell} d \tau \lesssim \int_{0}^{t}\langle t-\tau\rangle^{-\frac{\sigma_{1}+\sigma}{2}}\left\|u^{h}\right\|_{\dot{B}_{p, 1}^{\frac{d}{p}-\frac{d}{2}-\sigma_{1}}}\left\|\nabla a^{\ell}\right\|_{\dot{B}_{2,1}^{\frac{d}{p}}} d \tau \text {. }
$$

Observe that, as $\sigma_{1} \leq \sigma_{1}+\frac{d}{2}-\frac{d}{p}(p \geq 2)$, we have

$$
\left\|u^{h} \cdot \nabla a^{\ell}\right\|_{\dot{B}_{2, \infty}^{-\sigma_{1}}}^{\ell} \lesssim\left\|u^{h} \cdot \nabla a^{\ell}\right\|_{\dot{B}_{2, \infty}^{\frac{d}{p}-\frac{d}{2}-\sigma_{1}}}^{\ell} .
$$

Using the fact that $\frac{\sigma_{1}}{2}+\frac{d}{2 p}+1 \geq \frac{\sigma_{1}}{2}+\frac{d}{4}+\frac{1}{2}>1$ and $\frac{\sigma_{1}}{2}+\frac{d}{2 p}+1 \geq \frac{\sigma_{1}+\sigma}{2}$ for $p \leq d^{*}$ and $\sigma \leq \frac{d}{2}+1$ as well with (4.12), (4.13) and (4.2), we thus arrive at

$$
\begin{aligned}
\int_{0}^{t}\langle t-\tau\rangle^{-\frac{\sigma_{1}+\sigma}{2}}\left\|u^{h} \cdot \nabla a^{\ell}\right\|_{\dot{B}_{2, \infty}^{-\sigma_{1}}}^{\ell} d \tau & \lesssim \mathcal{D}_{p}^{2}(t) \int_{0}^{t}\langle t-\tau\rangle^{-\frac{\sigma_{1}+\sigma}{2}}\langle\tau\rangle^{-\frac{\sigma_{1}}{2}-\frac{d}{2 p}-1} d \tau \\
& \lesssim\langle t\rangle^{-\frac{\sigma_{1}+\sigma}{2}} \mathcal{D}_{p}^{2}(t) .
\end{aligned}
$$


The term $u^{h} \cdot \nabla u^{\ell}$ being completely similar to $u^{h} \cdot \nabla a^{\ell}$.

Let us finally handle the term corresponding to $g_{4}\left(a, u^{\ell}\right)$. Using (4.9), (4.3) and Proposition 3.4, we have

$$
\begin{aligned}
\int_{0}^{t}\langle t-\tau\rangle^{-\frac{\sigma_{1}+\sigma}{2}}\left\|g_{4}\left(a, u^{\ell}\right)\right\|_{\dot{B}_{2, \infty}^{-\sigma_{1}}}^{\ell} d \tau & \lesssim \int_{0}^{t}\langle t-\tau\rangle^{-\frac{\sigma_{1}+\sigma}{2}}\left\|g_{4}\left(a, u^{\ell}\right)\right\|_{\dot{B}_{2, \infty}^{\frac{d}{p}-\frac{d}{2}-\sigma_{1}}}^{\ell} d \tau \\
& \lesssim \int_{0}^{t}\langle t-\tau\rangle^{-\frac{\sigma_{1}+\sigma}{2}}\|\nabla a\|_{\dot{B}_{p, 1}^{\frac{d}{p}-\frac{d}{2}-\sigma_{1}}}\left\|\nabla u^{\ell}\right\|_{\dot{B}_{2,1}^{\frac{d}{p}}} d \tau
\end{aligned}
$$

where $\frac{d}{p}-\frac{d}{2}-\sigma_{1}+1 \geq 1-\frac{d}{p}>-\frac{d}{p}$ implies that $\frac{d}{p}-\frac{d}{2}-\sigma_{1}+1$ fullfils the regularity requirement in Proposition 3.4). Thanks to (4.12)-(4.13) and (4.2), we end up with

$$
\begin{aligned}
\int_{0}^{t}\langle t-\tau\rangle^{-\frac{\sigma_{1}+\sigma}{2}}\left\|g_{4}\left(a, u^{\ell}\right)\right\|_{\dot{B}_{2, \infty}^{-\sigma_{1}}}^{\ell} d \tau & \lesssim \mathcal{D}_{p}^{2}(t) \int_{0}^{t}\langle t-\tau\rangle^{-\frac{\sigma_{1}+\sigma}{2}}\langle\tau\rangle^{-\frac{\sigma_{1}}{2}-\frac{d}{2 p}-1} d \tau \\
& \lesssim\langle t\rangle^{-\frac{\sigma_{1}+\sigma}{2}} \mathcal{D}_{p}^{2}(t) .
\end{aligned}
$$

Hence, putting all estimates together lead to (4.7).

To bound nonlinear terms in $f^{h}$ and $g^{h}$, precisely,

$$
a \operatorname{div} u^{h}, \quad u \cdot \nabla a^{h}, u \cdot \nabla u^{h}, \quad k(a) \nabla a^{h}, \quad g_{3}\left(a, u^{h}\right) \text { and } g_{4}\left(a, u^{h}\right),
$$

we shall proceed those calculations differently depending on whether $2 \leq p \leq d$ or $p>d$.

Lemma 4.3. Let $2 \leq p \leq d$ and $d / p-d / 2<\sigma_{1} \leq \sigma_{0}$. If $p$ satisfies (2.2), then it holds that for all $t \geq 0$,

$$
\int_{0}^{t}\langle t-\tau\rangle^{-\frac{\sigma_{1}+\sigma}{2}}\left\|\left(f^{h}, g^{h}\right)(\tau)\right\|_{\dot{B}_{2, \infty}^{-\sigma_{1}}}^{\ell} d \tau \lesssim\langle t\rangle^{-\frac{\sigma_{1}+\sigma}{2}}\left(\mathcal{D}_{p}^{2}(t)+\mathcal{E}_{p}^{2}(t)\right) .
$$

Proof. We first deal with the case $\frac{d}{p}-\frac{d}{2}<\sigma_{1} \leq \sigma_{0}$ if $2 \leq p \leq d$. Owing to the embedding $L^{\frac{p}{2}} \hookrightarrow \dot{B}_{2, \infty}^{-\sigma_{0}}$ and Hölder inequality, one has

$$
\|F G\|_{\dot{B}_{2, \infty}^{-\sigma_{1}}}^{\ell} \lesssim\|F G\|_{\dot{B}_{2, \infty}^{-\sigma_{0}}}^{\ell} \lesssim\|F G\|_{L^{\frac{p}{2}}} \lesssim\|F\|_{L^{p}}\|G\|_{L^{p}}
$$

By Sobolev embedding properties, we obtain

$$
\|(a, u)\|_{L^{p}} \leq\left\|(a, u)^{\ell}\right\|_{L^{p}}+\left\|(a, u)^{h}\right\|_{L^{p}} \lesssim\|(a, u)\|_{\dot{B}_{2,1}^{\frac{d}{2}-\frac{d}{p}}}^{\ell}+\|(a, u)\|_{\dot{B}_{p, 1}^{\frac{d}{p}-1}}^{h} .
$$

Remembering that $-\sigma_{1}<\frac{d}{2}-\frac{d}{p}<\frac{d}{2}+1$ and $\alpha \geq \frac{\sigma_{1}}{2}+\frac{d}{4}-\frac{d}{2 p}$ for small enough $\varepsilon>0$ and using the definition of $\mathcal{D}_{p}(t)$, we get

$$
\|(a, u)(\tau)\|_{L^{p}} \lesssim\langle\tau\rangle^{-\frac{\sigma_{1}}{2}-\frac{d}{4}+\frac{d}{2 p}} \mathcal{D}_{p}(\tau),\left\|\nabla a^{h}(\tau)\right\|_{L^{p}} \lesssim\langle\tau\rangle^{-\alpha} \mathcal{D}_{p}(\tau) \text { for all } \tau \geq 0 .
$$

For the term with $u \cdot \nabla a^{h}$, we use (4.15) and (4.16), and get

$$
\begin{aligned}
\int_{0}^{t}\langle t-\tau\rangle^{-\frac{\sigma_{1}+\sigma}{2}}\left\|u \cdot \nabla a^{h}\right\|_{\dot{B}_{2, \infty}^{-\sigma_{1}}}^{\ell} d \tau & \lesssim \int_{0}^{t}\langle t-\tau\rangle^{-\frac{\sigma_{1}+\sigma}{2}}\|u\|_{L^{p}}\left\|\nabla a^{h}\right\|_{L^{p}} d \tau \\
& \lesssim \mathcal{D}_{p}^{2}(t) \int_{0}^{t}\langle t-\tau\rangle^{-\frac{\sigma_{1}+\sigma}{2}}\langle\tau\rangle^{-\frac{\sigma_{1}}{2}-\frac{d}{4}+\frac{d}{2 p}-\alpha} d \tau .
\end{aligned}
$$


The relation $\sigma \leq \frac{d}{2}+1$ implies that $\alpha \geq \frac{\sigma_{1}+\sigma}{2}$ for small enough $\varepsilon>0$. Consequently, we have

$$
\frac{\sigma_{1}}{2}+\frac{d}{4}-\frac{d}{2 p}+\alpha \geq \frac{\sigma_{1}+\sigma}{2} .
$$

Additionally, it is not hard to check that $\frac{\sigma_{1}}{2}+\frac{d}{4}-\frac{d}{2 p}+\alpha>1$. Therefore, inequality (4.2) implies that

$$
\int_{0}^{t}\langle t-\tau\rangle^{-\frac{\sigma_{1}+\sigma}{2}}\left\|u \cdot \nabla a^{h}\right\|_{\dot{B}_{2, \infty}^{-\sigma_{1}}}^{\ell} d \tau \lesssim\langle t\rangle^{-\frac{\sigma_{1}+\sigma}{2}} \mathcal{D}_{p}^{2}(t) .
$$

To handle the term with $k(a) \nabla a^{h}$, thanks to (4.15)-(4.16), we similarly arrive at

$$
\begin{aligned}
\int_{0}^{t}\langle t-\tau\rangle^{-\frac{\sigma_{1}+\sigma}{2}}\left\|k(a) \nabla a^{h}\right\|_{\dot{B}_{2, \infty}^{-\sigma_{1}}}^{\ell} d \tau & \lesssim \int_{0}^{t}\langle t-\tau\rangle^{-\frac{\sigma_{1}+\sigma}{2}}\|a\|_{L^{p}}\left\|\nabla a^{h}\right\|_{L^{p}} d \tau \\
& \lesssim \mathcal{D}_{p}^{2}(t) \int_{0}^{t}\langle t-\tau\rangle^{-\frac{\sigma_{1}+\sigma}{2}}\langle\tau\rangle^{-\frac{\sigma_{1}}{2}-\frac{d}{4}+\frac{d}{2 p}-\alpha} d \tau \\
& \lesssim\langle t\rangle^{-\frac{\sigma_{1}+\sigma}{2}} \mathcal{D}_{p}^{2}(t) .
\end{aligned}
$$

For the term with $a \operatorname{div} u^{h}$, we consider cases $t \geq 2$ and $t \leq 2$ separately. Then we use that, owing to (4.15), if $t \geq 2$,

$$
\begin{aligned}
\int_{0}^{t}\langle t-\tau\rangle^{-\frac{\sigma_{1}+\sigma}{2}}\left\|a \operatorname{div} u^{h}\right\|_{\dot{B}_{2, \infty}^{-\sigma_{1}}}^{\ell} d \tau & \lesssim \int_{0}^{t}\langle t-\tau\rangle^{-\frac{\sigma_{1}+\sigma}{2}}\|a\|_{L^{p}}\left\|\operatorname{div} u^{h}\right\|_{L^{p}} d \tau \\
& =\left(\int_{0}^{1}+\int_{1}^{t}\right)(\cdots) d \tau \triangleq I_{11}+I_{12} .
\end{aligned}
$$

Note that $\frac{d}{p}-1 \geq 0$, it follows from the definitions of $\mathcal{E}_{p}(t)$ and $\mathcal{D}_{p}(t)$ that

$$
I_{11} \lesssim\langle t\rangle^{-\frac{\sigma_{1}+\sigma}{2}}\left(\sup _{0 \leq \tau \leq 1}\|a\|_{L^{p}}\right) \int_{0}^{1}\|u\|_{\dot{B}_{p, 1}^{\frac{d}{p}}}^{h} d \tau \lesssim\langle t\rangle^{-\frac{\sigma_{1}+\sigma}{2}} \mathcal{D}_{p}(1) \mathcal{E}_{p}(1) .
$$

On the other hand, using (4.2), (4.16) and the fact that $\langle\tau\rangle \approx \tau$ as $\tau \geq 1$, one has for all $-\sigma_{1}<\sigma \leq \frac{d}{2}+1$,

$$
\begin{aligned}
I_{12}= & \int_{1}^{t}\langle t-\tau\rangle^{-\frac{\sigma_{1}+\sigma}{2}}\langle\tau\rangle^{-\frac{\sigma_{1}}{2}-\frac{d}{4}+\frac{d}{2 p}-\alpha}\left(\langle\tau\rangle^{\frac{\sigma_{1}}{2}+\frac{d}{4}-\frac{d}{2 p}}\|a\|_{L^{p}}\right)\left(\tau^{\alpha}\left\|\operatorname{div} u^{h}\right\|_{L^{p}}\right) d \tau \\
\lesssim & \left(\sup _{1 \leq \tau \leq t}\langle\tau\rangle^{\frac{\sigma_{1}}{2}+\frac{d}{4}-\frac{d}{2 p}}\|a\|_{L^{p}}\right)\left(\sup _{1 \leq \tau \leq t} \tau^{\alpha}\|u\|_{\dot{B}_{p, 1}^{\frac{d}{p}}}^{h}\right) \\
& \times \int_{1}^{t}\langle t-\tau\rangle^{-\frac{\sigma_{1}+\sigma}{2}}\langle\tau\rangle^{-\frac{\sigma_{1}}{2}-\frac{d}{4}+\frac{d}{2 p}-\alpha} d \tau \lesssim\langle t\rangle^{-\frac{\sigma_{1}+\sigma}{2}} \mathcal{D}_{p}^{2}(t) .
\end{aligned}
$$

Therefore, we deduce that

$$
\int_{0}^{t}\langle t-\tau\rangle^{-\frac{\sigma_{1}+\sigma}{2}}\left\|a \operatorname{div} u^{h}\right\|_{\dot{B}_{2, \infty}^{-\sigma_{1}}}^{\ell} d \tau \lesssim\langle t\rangle^{-\frac{\sigma_{1}+\sigma}{2}}\left(\mathcal{D}_{p}(t) \mathcal{E}_{p}(t)+\mathcal{D}_{p}^{2}(t)\right) \text { for all } t \geq 2 .
$$

In the trivial case $t \leq 2$, we have $\langle t\rangle \approx 1$ and $\langle t-\tau\rangle \approx 1$ for $0 \leq \tau \leq t \leq 2$, and

$$
\int_{0}^{t}\langle t-\tau\rangle^{-\frac{\sigma_{1}+\sigma}{2}}\left\|a \operatorname{div} u^{h}\right\|_{\dot{B}_{2, \infty}^{-\sigma_{1}}}^{\ell} d \tau \lesssim\|a\|_{L_{t}^{\infty}\left(L^{p}\right)}\left\|u^{h}\right\|_{L_{t}^{1}\left(\dot{B}_{p, 1}^{\frac{d}{p}}\right)} \lesssim \mathcal{D}_{p}(t) \mathcal{E}_{p}(t) .
$$

Those terms with $u \cdot \nabla u^{h}$ and $g_{3}\left(a, u^{h}\right)$ may be treated along the same lines. 
Finally, in order to bound the term with $g_{4}\left(a, u^{h}\right)$, we use (4.15) and get if $t \geq 2$,

$$
\begin{aligned}
\int_{0}^{t}\langle t-\tau\rangle^{-\frac{\sigma_{1}+\sigma}{2}}\left\|g_{4}\left(a, u^{h}\right)\right\|_{\dot{B}_{2, \infty}^{-\sigma_{1}}}^{\ell} d \tau & \lesssim \int_{0}^{t}\langle t-\tau\rangle^{-\frac{\sigma_{1}+\sigma}{2}}\|\nabla a\|_{L^{p}}\left\|\nabla u^{h}\right\|_{L^{p}} d \tau \\
& =\left(\int_{0}^{1}+\int_{1}^{t}\right)(\cdots) d \tau \triangleq J_{11}+J_{12} .
\end{aligned}
$$

Note that $-\sigma_{1}<\frac{d}{2}-\frac{d}{p}+1<\frac{d}{2}+1$ and $\alpha \geq \frac{\sigma_{1}}{2}+\frac{d}{4}-\frac{d}{2 p}+\frac{1}{2}>0$ for small enough $\varepsilon>0$, we have

$$
\|\nabla a(\tau)\|_{L^{p}} \lesssim\|a(\tau)\|_{\dot{B}_{2,1}^{\frac{d}{2}-\frac{d}{p}+1}}^{\ell}+\|a\|_{\dot{B}_{p, 1}^{\frac{d}{p}}}^{h} \lesssim\langle\tau\rangle^{-\frac{\sigma_{1}}{2}-\frac{d}{4}+\frac{d}{2 p}-\frac{1}{2}} \mathcal{D}_{p}(\tau) \text { for all } \tau \geq 0 .
$$

Hence using the embedding and the definitions of $\mathcal{E}_{p}(t)$ and $\mathcal{D}_{p}(t)$ yields

$$
J_{11} \lesssim\langle t\rangle^{-\frac{\sigma_{1}+\sigma}{2}}\left(\sup _{0 \leq \tau \leq 1}\|\nabla a\|_{L^{p}}\right) \int_{0}^{1}\|u\|_{\dot{B}_{p, 1}^{\frac{d}{p}}}^{h} d \tau \lesssim\langle t\rangle^{-\frac{\sigma_{1}+\sigma}{2}} \mathcal{D}_{p}(1) \mathcal{E}_{p}(1) .
$$

Thanks to (4.2), we conclude that

$$
\begin{aligned}
J_{12} \lesssim & \left(\sup _{1 \leq \tau \leq t}\langle\tau\rangle^{\frac{\sigma_{1}}{2}+\frac{d}{4}-\frac{d}{2 p}+\frac{1}{2}}\|\nabla a\|_{L^{p}}\right)\left(\sup _{1 \leq \tau \leq t} \tau^{\alpha}\|u\|_{\dot{B}_{p, 1}^{\frac{d}{p}}}^{h}\right) \\
& \times \int_{1}^{t}\langle t-\tau\rangle^{-\frac{\sigma_{1}+\sigma}{2}}\langle\tau\rangle^{-\frac{\sigma_{1}}{2}-\frac{d}{4}+\frac{d}{2 p}-\frac{1}{2}-\alpha} d \tau \lesssim\langle t\rangle^{-\frac{\sigma_{1}+\sigma}{2}} \mathcal{D}_{p}^{2}(t) .
\end{aligned}
$$

Therefore, we end up with

$$
\int_{0}^{t}\langle t-\tau\rangle^{-\frac{\sigma_{1}+\sigma}{2}}\left\|g_{4}\left(a, u^{h}\right)\right\|_{\dot{B}_{2, \infty}^{-\sigma_{1}}}^{\ell} d \tau \lesssim\langle t\rangle^{-\frac{\sigma_{1}+\sigma}{2}}\left(\mathcal{D}_{p}(t) \mathcal{E}_{p}(t)+\mathcal{D}_{p}^{2}(t)\right) \text { for all } t \geq 2 .
$$

The case $t \leq 2$ implies that $\langle t\rangle \approx 1$ and $\langle t-\tau\rangle \approx 1$ for $0 \leq \tau \leq t \leq 2$, so we get

$$
\int_{0}^{t}\langle t-\tau\rangle^{-\frac{\sigma_{1}+\sigma}{2}}\left\|g_{4}\left(a, u^{h}\right)\right\|_{\dot{B}_{2, \infty}^{-\sigma_{1}}}^{\ell} d \tau \lesssim\|\nabla a\|_{L_{t}^{\infty}\left(L^{p}\right)}\left\|u^{h}\right\|_{L_{t}^{1}\left(\dot{B}_{p, 1}^{\frac{d}{p}}\right)} \lesssim \mathcal{D}_{p}(t) \mathcal{E}_{p}(t) .
$$

In summary, the time-weighted inequality (4.14) is followed.

Lemma 4.4. Let $2 \leq p \leq d$ and $1-d / 2<\sigma_{1} \leq d / p-d / 2$. If $p$ satisfies (2.2), then it holds that for all $t \geq 0$,

$$
\int_{0}^{t}\langle t-\tau\rangle^{-\frac{\sigma_{1}+\sigma}{2}}\left\|\left(f^{h}, g^{h}\right)(\tau)\right\|_{\dot{B}_{2, \infty}^{-\sigma_{1}}}^{\ell} d \tau \lesssim\langle t\rangle^{-\frac{\sigma_{1}+\sigma}{2}}\left(\mathcal{D}_{p}^{2}(t)+\mathcal{E}_{p}^{2}(t)\right)
$$

Proof. We handle another case $1-\frac{d}{2}<\sigma_{1} \leq \frac{d}{p}-\frac{d}{2}$ if $2 \leq p \leq d$. By Sobolev embedding properties and Hölder inequality, we have

$$
\begin{aligned}
& \left\|F G^{h}\right\|_{\dot{B}_{2, \infty}^{-\sigma_{1}}}^{\ell} \lesssim\left\|F G^{h}\right\|_{\dot{B}_{2, \infty}^{0}}^{\ell} \lesssim\left\|F G^{h}\right\|_{L^{2}} \lesssim\|F\|_{L^{p^{*}}}\left\|G^{h}\right\|_{L^{p}} \lesssim\|F\|_{\dot{B}_{p, 1}^{\sigma_{0}}}\|G\|_{\dot{B}_{p, 1}^{\frac{d}{p}-1}}^{h}, \\
& \left\|F G^{h}\right\|_{\dot{B}_{2, \infty}^{-\sigma_{1}}}^{\ell} \lesssim\left\|F G^{h}\right\|_{\dot{B}_{2, \infty}^{0}}^{\ell} \lesssim\left\|F G^{h}\right\|_{L^{2}} \lesssim\|F\|_{L^{d}}\left\|G^{h}\right\|_{L^{d^{*}}} \lesssim\|F\|_{\dot{B}_{p, 1}^{\frac{d}{p}-1}\|G\|_{\dot{B}_{p, 1}^{\frac{d}{p}}}^{h},},
\end{aligned}
$$

where $1 / p+1 / p^{*}=1 / d+1 / d^{*}=1 / 2$. In addition, we also get, due to the embedding $\dot{B}_{2,1}^{\frac{d}{p}} \hookrightarrow \dot{B}_{p, 1}^{\sigma_{0}}$

$$
\left\|\left(a, u^{\ell}\right)(\tau)\right\|_{\dot{B}_{p, 1}^{\sigma_{0}}} \lesssim\|(a, u)(\tau)\|_{\dot{B}_{2,1}^{\frac{d}{p}}}^{\ell}+\|a(\tau)\|_{\dot{B}_{p, 1}^{\frac{d}{p}}}^{h} \lesssim\langle\tau\rangle^{-\frac{\sigma_{1}}{2}-\frac{d}{2 p}} \mathcal{D}_{p}(\tau),
$$


where we noticed the fact that $-\sigma_{1}<\frac{d}{2}-1 \leq \frac{d}{p}<\frac{d}{2}+1$ for $p \leq d^{*}$, and that $\alpha \geq \frac{\sigma_{1}}{2}+\frac{d}{2 p}$ for small enough $\varepsilon>0$.

To bound the term with $a \operatorname{div} u^{h}$, we take advantage of (4.18) and get, if $t \geq 2$,

$$
\begin{aligned}
\int_{0}^{t}\langle t-\tau\rangle^{-\frac{\sigma_{1}+\sigma}{2}}\left\|a \operatorname{div} u^{h}\right\|_{\dot{B}_{2, \infty}^{-\sigma_{1}}}^{\ell} d \tau & \lesssim \int_{0}^{t}\langle t-\tau\rangle^{-\frac{\sigma_{1}+\sigma}{2}}\|a\|_{\dot{B}_{p, 1}^{\sigma_{0}}}\|\operatorname{div} u\|_{\dot{B}_{p, 1}^{\frac{d}{p}-1}}^{h} d \tau \\
& =\left(\int_{0}^{1}+\int_{1}^{t}\right)(\cdots) d \tau \triangleq I_{21}+I_{22} .
\end{aligned}
$$

With the aid of (4.20), one can obtain

$$
I_{21} \lesssim\langle t\rangle^{-\frac{\sigma_{1}+\sigma}{2}}\left(\sup _{0 \leq \tau \leq 1}\|a\|_{\dot{B}_{p, 1}^{\sigma_{0}}}\right) \int_{0}^{1}\|u\|_{\dot{B}_{p, 1}^{\frac{d}{p}}}^{h} d \tau \lesssim\langle t\rangle^{-\frac{\sigma_{1}+\sigma}{2}} \mathcal{D}_{p}(1) \mathcal{E}_{p}(1) .
$$

By using (4.2) and (4.20), we conclude that for all $-\sigma_{1}<\sigma \leq \frac{d}{2}+1$,

$$
\begin{aligned}
I_{22}= & \int_{1}^{t}\langle t-\tau\rangle^{-\frac{\sigma_{1}+\sigma}{2}}\langle\tau\rangle^{-\frac{\sigma_{1}}{2}-\frac{d}{2 p}-\alpha}\left(\langle\tau\rangle^{\frac{\sigma_{1}}{2}+\frac{d}{2 p}}\|a\|_{\dot{B}_{p, 1}^{\sigma_{0}}}\right)\left(\tau^{\alpha}\|\operatorname{div} u\|_{\dot{B}_{p, 1}^{\frac{d}{p}-1}}^{h}\right) d \tau \\
\lesssim & \left(\sup _{1 \leq \tau \leq t}\langle\tau\rangle^{\frac{\sigma_{1}}{2}+\frac{d}{2 p}}\|a\|_{\dot{B}_{p, 1}^{\sigma_{0}}}\right)\left(\sup _{1 \leq \tau \leq t} \tau^{\alpha}\|u\|_{\dot{B}_{p, 1}^{\frac{d}{p}}}^{h}\right) \\
& \times \int_{1}^{t}\langle t-\tau\rangle^{-\frac{\sigma_{1}+\sigma}{2}}\langle\tau\rangle^{-\frac{\sigma_{1}}{2}-\frac{d}{2 p}-\alpha} d \tau \lesssim\langle t\rangle^{-\frac{\sigma_{1}+\sigma}{2}} \mathcal{D}_{p}^{2}(t),
\end{aligned}
$$

where we noticed that $\frac{\sigma_{1}}{2}+\frac{d}{2 p}+\alpha>1$ and that $\frac{\sigma_{1}}{2}+\frac{d}{2 p}+\alpha \geq \frac{\sigma_{1}+\sigma}{2}$ for small enough $\varepsilon>0$ and $\sigma \leq \frac{d}{2}+1$. Therefore, we obtain

$$
\int_{0}^{t}\langle t-\tau\rangle^{-\frac{\sigma_{1}+\sigma}{2}}\left\|a \operatorname{div} u^{h}\right\|_{\dot{B}_{2, \infty}^{-\sigma_{1}}}^{\ell} d \tau \lesssim\langle t\rangle^{-\frac{\sigma_{1}+\sigma}{2}}\left(\mathcal{D}_{p}(t) \mathcal{E}_{p}(t)+\mathcal{D}_{p}^{2}(t)\right) \text { for all } t \geq 2 .
$$

In the case $t \leq 2$, we have $\langle t\rangle \approx 1$ and $\langle t-\tau\rangle \approx 1$ for $0 \leq \tau \leq t \leq 2$, and

$$
\int_{0}^{t}\langle t-\tau\rangle^{-\frac{\sigma_{1}+\sigma}{2}}\left\|a \operatorname{div} u^{h}\right\|_{\dot{B}_{2, \infty}^{-\sigma_{1}}}^{\ell} d \tau \lesssim\|a\|_{L_{t}^{\infty}\left(\dot{B}_{p, 1}^{\sigma_{0}}\right)}\left\|u^{h}\right\|_{L_{t}^{1}\left(\dot{B}_{p, 1}^{\frac{d}{p}}\right)} \lesssim \mathcal{D}_{p}(t) \mathcal{E}_{p}(t) .
$$

Regarding the term with $u \cdot \nabla a^{h}$, we write that $u \cdot \nabla a^{h}=u^{\ell} \cdot \nabla a^{h}+u^{h} \cdot \nabla a^{h}$. To handle the term containing $u^{\ell} \cdot \nabla a^{h}$, we use the following inequality, owing to the relation $\frac{d}{p}-1 \geq 0$ and the definition of $\mathcal{D}_{p}(t)$,

$$
\|\nabla a(\tau)\|_{\dot{B}_{p, 1}^{\frac{d}{p}-1}}^{h} \lesssim\langle\tau\rangle^{-\alpha} \mathcal{D}_{p}(\tau),
$$

together with (4.18), (4.20) and (4.2), we thus get

$$
\begin{aligned}
\int_{0}^{t}\langle t-\tau\rangle^{-\frac{\sigma_{1}+\sigma}{2}}\left\|u^{\ell} \cdot \nabla a^{h}\right\|_{\dot{B}_{2, \infty}^{-\sigma_{1}}}^{\ell} d \tau & \lesssim \int_{0}^{t}\langle t-\tau\rangle^{-\frac{\sigma_{1}+\sigma}{2}}\left\|u^{\ell}\right\|_{\dot{B}_{p, 1}^{\sigma_{0}}}\|\nabla a\|_{\dot{B}_{p, 1}^{\frac{d}{p}-1}}^{h} d \tau \\
& \lesssim \mathcal{D}_{p}^{2}(t) \int_{0}^{t}\langle t-\tau\rangle^{-\frac{\sigma_{1}+\sigma}{2}}\langle\tau\rangle^{-\frac{\sigma_{1}}{2}-\frac{d}{2 p}-\alpha} d \tau \\
& \lesssim\langle t\rangle^{-\frac{\sigma_{1}+\sigma}{2}} \mathcal{D}_{p}^{2}(t) .
\end{aligned}
$$


To bound the term with $u^{h} \cdot \nabla a^{h}$, we have by using (4.18) and the relation $\sigma_{0} \leq \frac{d}{p}$,

$$
\begin{aligned}
\int_{0}^{t}\langle t-\tau\rangle^{-\frac{\sigma_{1}+\sigma}{2}}\left\|u^{h} \cdot \nabla a^{h}\right\|_{\dot{B}_{2, \infty}^{-\sigma_{1}}}^{\ell} d \tau & \lesssim \int_{0}^{t}\langle t-\tau\rangle^{-\frac{\sigma_{1}+\sigma}{2}}\left\|u^{h}\right\|_{\dot{B}_{p, 1}^{\frac{d}{p}}}\|\nabla a\|_{\dot{B}_{p, 1}^{\frac{d}{p}-1}}^{h} d \tau \\
& =\left(\int_{0}^{1}+\int_{1}^{t}\right)(\cdots) d \tau \triangleq J_{21}+J_{22} .
\end{aligned}
$$

It is clear that $J_{21} \lesssim\langle t\rangle^{-\frac{\sigma_{1}+\sigma}{2}} \mathcal{E}_{p}^{2}(1)$ and that, owing to (4.2) and (4.23), if $t \geq 1$,

$$
\begin{aligned}
J_{22} & =\int_{1}^{t}\langle t-\tau\rangle^{-\frac{\sigma_{1}+\sigma}{2}}\langle\tau\rangle^{-2 \alpha}\left(\tau^{\alpha}\left\|u^{h}\right\|_{\dot{B}_{p, 1}^{\frac{d}{p}}}\right)\left(\langle\tau\rangle^{\alpha}\|\nabla a\|_{\dot{B}_{p, 1}^{\frac{d}{p}-1}}^{h}\right) d \tau \\
& \lesssim\left(\sup _{1 \leq \tau \leq t} \tau^{\alpha}\|u\|_{\dot{B}_{p, 1}^{\frac{d}{p}}}^{h}\right)\left(\sup _{1 \leq \tau \leq t}\langle\tau\rangle^{\alpha}\|a\|_{\dot{B}_{p, 1}^{\frac{d}{p}}}^{h}\right) \int_{1}^{t}\langle t-\tau\rangle^{-\frac{\sigma_{1}+\sigma}{2}}\langle\tau\rangle^{-2 \alpha} d \tau \\
& \lesssim\langle t\rangle^{-\frac{\sigma_{1}+\sigma}{2}} \mathcal{D}_{p}^{2}(t),
\end{aligned}
$$

since $\alpha \geq \frac{\sigma_{1}}{2}+\frac{d}{2 p}$ for small enough $\varepsilon>0$.

For the term with $k(a) \nabla a^{h}$, we use (4.18), (4.3) and Proposition 3.4 with $\sigma_{0}>-\frac{d}{p}$, and get

$$
\left\|k(a) \nabla a^{h}\right\|_{\dot{B}_{2, \infty}^{-\sigma_{1}}}^{\ell} \lesssim\|k(a)\|_{\dot{B}_{p, 1}^{\sigma_{0}}}^{\ell}\|\nabla a\|_{\dot{B}_{p, 1}^{\frac{d}{p}-1}}^{h} \lesssim\|a\|_{\dot{B}_{p, 1}^{\sigma_{0}}}\|a\|_{\dot{B}_{p, 1}^{\frac{d}{p}}}^{h}
$$

Furthermore, by using (4.20), (4.23) and (4.2), we end up with

$$
\begin{aligned}
\int_{0}^{t}\langle t-\tau\rangle^{-\frac{\sigma_{1}+\sigma}{2}}\left\|k(a) \nabla a^{h}\right\|_{\dot{B}_{2, \infty}^{-\sigma_{1}}}^{\ell} d \tau & \lesssim \int_{0}^{t}\langle t-\tau\rangle^{-\frac{\sigma_{1}+\sigma}{2}}\|a\|_{\dot{B}_{p, 1}^{\sigma_{0}}}\|a\|_{\dot{B}_{p, 1}^{\frac{d}{p}}}^{h} d \tau \\
& \lesssim \mathcal{D}_{p}^{2}(t) \int_{0}^{t}\langle t-\tau\rangle^{-\frac{\sigma_{1}+\sigma}{2}}\langle\tau\rangle^{-\frac{\sigma_{1}}{2}-\frac{d}{2 p}-\alpha} d \tau \\
& \lesssim\langle t\rangle^{-\frac{\sigma_{1}+\sigma}{2}} \mathcal{D}_{p}^{2}(t) .
\end{aligned}
$$

For the term with $g_{3}\left(a, u^{h}\right)$, by repeating the procedure leading to (4.21) and (4.22), we conclude that

$\int_{0}^{t}\langle t-\tau\rangle^{-\frac{\sigma_{1}+\sigma}{2}}\left\|g_{3}\left(a, u^{h}\right)\right\|_{\dot{B}_{2, \infty}^{-\sigma_{1}}}^{\ell} d \tau \lesssim\langle t\rangle^{-\frac{\sigma_{1}+\sigma}{2}}\left(\mathcal{D}_{p}(t) \mathcal{E}_{p}(t)+\mathcal{D}_{p}^{2}(t)\right)$ for $-\sigma_{1}<\sigma \leq \frac{d}{2}+1$.

To handle the term involving $u \cdot \nabla u^{h}$, we write that, thanks to (4.19)

$$
\begin{aligned}
\int_{0}^{t}\langle t-\tau\rangle^{-\frac{\sigma_{1}+\sigma}{2}}\left\|u \cdot \nabla u^{h}\right\|_{\dot{B}_{2, \infty}^{-\sigma_{1}}}^{\ell} d \tau & \lesssim \int_{0}^{t}\langle t-\tau\rangle^{-\frac{\sigma_{1}+\sigma}{2}}\|u\|_{\dot{B}_{p, 1}^{\frac{d}{p}-1}}\|\nabla u\|_{\dot{B}_{p, 1}^{\frac{d}{p}}}^{h} d \tau \\
& =\left(\int_{0}^{1}+\int_{1}^{t}\right)(\cdots) d \tau \triangleq K_{21}+K_{22} .
\end{aligned}
$$

It following from the definition of $\mathcal{E}(t)$ that

$$
K_{21} \lesssim\langle t\rangle^{-\frac{\sigma_{1}+\sigma}{2}} \mathcal{E}_{p}^{2}(1)
$$


Using the relation $-\sigma_{1}<\frac{d}{2}-1<\frac{d}{2}+1$ and $\alpha \geq \frac{\sigma_{1}}{2}+\frac{d}{4}-\frac{1}{2}$ for small enough $\varepsilon>0$ indicates that

$$
\|u\|_{\dot{B}_{p, 1}^{\frac{d}{p}-1}} \lesssim\|u\|_{\dot{B}_{2,1}^{\frac{d}{2}-1}}^{\ell}+\|u\|_{\dot{B}_{p, 1}^{\frac{d}{p}-1}}^{h} \lesssim\langle\tau\rangle^{-\frac{\sigma_{1}}{2}-\frac{d}{4}+\frac{1}{2}} \mathcal{D}_{p}(\tau)
$$

together with (4.2), we thus get, if $t \geq 1$,

$$
\begin{aligned}
K_{22} \lesssim & \int_{1}^{t}\langle t-\tau\rangle^{-\frac{\sigma_{1}+\sigma}{2}}\langle\tau\rangle^{-\frac{\sigma_{1}}{2}-\frac{d}{4}+\frac{1}{2}-\alpha}\left(\langle\tau\rangle^{\frac{\sigma_{1}}{2}+\frac{d}{4}-\frac{1}{2}}\|u\|_{\dot{B}_{p, 1}^{\frac{d}{p}-1}}\right)\left(\tau^{\alpha}\|\nabla u\|_{\dot{B}_{p, 1}^{\frac{d}{p}}}^{h}\right) d \tau \\
\lesssim & \left(\sup _{1 \leq \tau \leq t}\langle\tau\rangle^{\frac{\sigma_{1}}{2}+\frac{d}{4}-\frac{1}{2}}\|u\|_{\dot{B}_{p, 1}^{\frac{d}{p}-1}}\right)\left(\sup _{1 \leq \tau \leq t} \tau^{\alpha}\|\nabla u\|_{\dot{B}_{p, 1}^{\frac{d}{p}}}^{h}\right) \\
& \times \int_{1}^{t}\langle t-\tau\rangle^{-\frac{\sigma_{1}+\sigma}{2}}\langle\tau\rangle^{-\frac{\sigma_{1}}{2}-\frac{d}{4}+\frac{1}{2}-\alpha} d \tau \lesssim\langle t\rangle^{-\frac{\sigma_{1}+\sigma}{2}} \mathcal{D}_{p}^{2}(t),
\end{aligned}
$$

since the relations $\sigma_{1}>1-\frac{d}{2}$ and $\alpha>1$ lead to $\frac{\sigma_{1}}{2}+\frac{d}{4}-\frac{1}{2}+\alpha>1$ and $\frac{\sigma_{1}}{2}+\frac{d}{4}-\frac{1}{2}+\alpha \geq$ $\frac{\sigma_{1}+\sigma}{2}$ for $\sigma \leq \frac{d}{2}+1$.

Let us finally look at the term with $g_{4}\left(a, u^{h}\right)$. We observe that, owing to (4.19) and Proposition 3.4,

$$
\begin{aligned}
\int_{0}^{t}\langle t-\tau\rangle^{-\frac{\sigma_{1}+\sigma}{2}}\left\|g_{4}\left(a, u^{h}\right)\right\|_{\dot{B}_{2, \infty}^{-\sigma_{1}}}^{\ell} d \tau & \lesssim \int_{0}^{t}\langle t-\tau\rangle^{-\frac{\sigma_{1}+\sigma}{2}}\|a\|_{\dot{B}_{p, 1}^{\frac{d}{p}}}\|\nabla u\|_{\dot{B}_{p, 1}^{\frac{d}{p}}}^{h} d \tau \\
& =\left(\int_{0}^{1}+\int_{1}^{t}\right)(\cdots) d \tau \triangleq L_{21}+L_{22} .
\end{aligned}
$$

It is clear that $L_{21} \lesssim\langle t\rangle^{-\frac{\sigma_{1}+\sigma}{2}} \mathcal{E}_{p}^{2}(1)$ and that, owing to (4.2) and (4.10), if $t \geq 1$,

$$
\begin{aligned}
L_{22}= & \int_{1}^{t}\langle t-\tau\rangle^{-\frac{\sigma_{1}+\sigma}{2}}\langle\tau\rangle^{-\frac{\sigma_{1}}{2}-\frac{d}{2}-\alpha}\left(\langle\tau\rangle^{\frac{\sigma_{1}}{2}+\frac{d}{4}}\|a\|_{\dot{B}_{p, 1}^{\frac{d}{p}}}\right)\left(\tau^{\alpha}\|\nabla u\|_{\dot{B}_{p, 1}^{\frac{d}{p}}}^{h}\right) d \tau \\
\lesssim & \left(\sup _{1 \leq \tau \leq t}\langle\tau\rangle^{\frac{\sigma_{1}}{2}+\frac{d}{4}}\|a\|_{\dot{B}_{p, 1}^{\frac{d}{p}}}\right)\left(\sup _{1 \leq \tau \leq t} \tau^{\alpha}\|u\|_{\dot{B}_{p, 1}^{\frac{d}{p}+1}}^{h}\right) \\
& \times \int_{1}^{t}\langle t-\tau\rangle^{-\frac{\sigma_{1}+\sigma}{2}}\langle\tau\rangle^{-\frac{\sigma_{1}}{2}-\frac{d}{4}-\alpha} d \tau \lesssim\langle t\rangle^{-\frac{\sigma_{1}+\sigma}{2}} \mathcal{D}_{p}^{2}(t) .
\end{aligned}
$$

Therefore, the proof of Lemma 4.4 is complete.

Lemma 4.5. Let $p$ satisfy $(2.2)$ and $p>d$. It holds that for all $t \geq 0$,

$$
\int_{0}^{t}\langle t-\tau\rangle^{-\frac{\sigma_{1}+\sigma}{2}}\left\|\left(f^{h}, g^{h}\right)(\tau)\right\|_{\dot{B}_{2, \infty}^{-\sigma_{1}}}^{\ell} d \tau \lesssim\langle t\rangle^{-\frac{\sigma_{1}+\sigma}{2}}\left(\mathcal{D}_{p}^{2}(t)+\mathcal{E}_{p}^{2}(t)\right) .
$$

Proof. Let us end the first step in the case where (2.2) is fulfilled and $p>d$. Using the fact that $\sigma_{1} \leq \sigma_{0}$ and applying (3.5) with $s=1-\frac{d}{p}$ yield

$$
\left\|F G^{h}\right\|_{\dot{B}_{2, \infty}^{-\sigma_{1}}}^{\ell} \lesssim\left\|F G^{h}\right\|_{\dot{B}_{2, \infty}^{-\sigma_{0}}}^{\ell} \lesssim\left(\|F\|_{\dot{B}_{p, 1}^{1-\frac{d}{p}}}+\left\|\dot{S}_{j_{0}+N_{0}} F\right\|_{L^{p^{*}}}\right)\left\|G^{h}\right\|_{\dot{B}_{p, 1}^{\frac{d}{p}-1}}
$$


with $\frac{1}{p^{*}} \triangleq \frac{1}{2}-\frac{1}{p}$ and thus, because $\dot{B}_{2,1}^{\frac{d}{p}} \hookrightarrow L^{p^{*}}$,

$$
\left\|F G^{h}\right\|_{\dot{B}_{2, \infty}^{-\sigma_{1}}}^{\ell} \lesssim\left\|F G^{h}\right\|_{\dot{B}_{2, \infty}^{-\sigma_{0}}}^{\ell} \lesssim\left(\left\|F^{\ell}\right\|_{\dot{B}_{2,1}^{\frac{d}{p}}}+\|F\|_{\dot{B}_{p, 1}^{1-\frac{d}{p}}}\right)\left\|G^{h}\right\|_{\dot{B}_{p, 1}^{\frac{d}{p}-1}} .
$$

On the other hand, we claim that

$$
\left\|\left(a, u^{\ell}\right)(\tau)\right\|_{\dot{B}_{p, 1}^{1-\frac{d}{p}}} \lesssim\langle\tau\rangle^{-\frac{\sigma_{1}+1-\sigma_{0}}{2}} \mathcal{D}_{p}(\tau)
$$

and also that

$$
\left\|(a, u)^{\ell}(\tau)\right\|_{\dot{B}_{2,1}^{\frac{d}{p}}} \lesssim\langle\tau\rangle^{-\frac{\sigma_{1}}{2}-\frac{d}{2 p}} \mathcal{D}_{p}(\tau) \text { for all } \tau \geq 0 .
$$

Indeed, owing to the embedding and the definition of $\mathcal{D}_{p}(t)$, we have

$$
\left\|(a, u)^{\ell}(\tau)\right\|_{\dot{B}_{p, 1}^{1-\frac{d}{p}}} \lesssim\left\|(a, u)^{\ell}(\tau)\right\|_{\dot{B}_{2,1}^{1-\sigma_{0}}} \lesssim\langle\tau\rangle^{-\frac{\sigma_{1}+1-\sigma_{0}}{2}} \mathcal{D}_{p}(\tau) .
$$

Here, we used the relation $p>d$ which indicates that $-\sigma_{1}<\frac{d}{2}-1<1-\sigma_{0}<\frac{d}{2}+1$.

As $1-\frac{d}{p}<\frac{d}{p}$, it holds that

$$
\left\|a^{h}(\tau)\right\|_{\dot{B}_{p, 1}^{1-\frac{d}{p}}} \lesssim\left\|a^{h}(\tau)\right\|_{\dot{B}_{p, 1}^{\frac{d}{p}}} \lesssim\langle\tau\rangle^{-\alpha} \mathcal{D}_{p}(\tau),
$$

and (4.28) is thus fulfilled by $a^{h}$ due to $\frac{\sigma_{1}+1-\sigma_{0}}{2}<\frac{\sigma_{1}}{2}+\frac{d}{4}+\frac{1}{2} \leq \alpha$ for small enough $\varepsilon>0$. Thanks to $-\sigma_{1}<\frac{d}{2}-1 \leq \frac{d}{p}<\frac{d}{2}+1$ for $p \leq d^{*}$, the inequality (4.29) is easily followed by the definition of $\mathcal{D}_{p}(t)$.

Note also that if $p>d$ and $p$ fulfills (2.2), then we have by interpolation

$$
\left\|u^{h}(\tau)\right\|_{\dot{B}_{p, 1}^{1-\frac{d}{p}}} \lesssim\left\|u^{h}(\tau)\right\|_{\dot{B}_{p, 1}^{\frac{d}{p}-1}}^{\frac{d}{p}}\left\|u^{h}(\tau)\right\|_{\dot{B}_{p, 1}^{\frac{d}{p}+1}}^{1-\frac{d}{p}} .
$$

Consequently, it follows from the definition of $\mathcal{D}_{p}(t)$ that

$$
\left\|u^{h}(\tau)\right\|_{\dot{B}_{p, 1}^{1-\frac{d}{p}}} \lesssim \tau^{-\left(1-\frac{d}{p}\right) \alpha}\langle\tau\rangle^{-\frac{d}{p} \alpha} \mathcal{D}_{p}(\tau) \text { for all } \tau \geq 0
$$

Here, it is easy to check that

$$
0<\left(1-\frac{d}{p}\right) \alpha<\left(1-\frac{d}{p}\right)\left(\frac{2 d}{p}+\frac{1}{2}\right)=1-\frac{d}{2 p}-2\left(\frac{d}{p}-\frac{1}{2}\right)^{2}<1 .
$$

Taking $F=u$ and $G^{h}=\nabla a^{h}$ in (4.27) and using (4.28), (4.29) and (4.30), we get

$$
\begin{aligned}
& \int_{0}^{t}\langle t-\tau\rangle^{-\frac{\sigma_{1}+\sigma}{2}}\left\|u \cdot \nabla a^{h}\right\|_{\dot{B}_{2, \infty}^{-\sigma_{1}}}^{\ell} d \tau \\
\lesssim & \int_{0}^{t}\langle t-\tau\rangle^{-\frac{\sigma_{1}+\sigma}{2}}\left(\left\|u^{\ell}\right\|_{\dot{B}_{2,1}^{\frac{d}{p}}}+\left\|u^{\ell}\right\|_{\dot{B}_{p, 1}^{1-\frac{d}{p}}}+\left\|u^{h}\right\|_{\dot{B}_{p, 1}^{1-\frac{d}{p}}}\right)\left\|\nabla a^{h}\right\|_{\dot{B}_{p, 1}^{\frac{d}{p}-1}} d \tau \\
\lesssim & \mathcal{D}_{p}^{2}(t) \int_{0}^{t}\langle t-\tau\rangle^{-\frac{\sigma_{1}+\sigma}{2}}\left(\langle\tau\rangle^{-\frac{\sigma_{1}}{2}-\frac{d}{2 p}}+\langle\tau\rangle^{-\frac{\sigma_{1}+1-\sigma_{0}}{2}}+\tau^{-\left(1-\frac{d}{p}\right) \alpha}\langle\tau\rangle^{-\frac{d}{p} \alpha}\right)\langle\tau\rangle^{-\alpha} d \tau .
\end{aligned}
$$

Keeping in mind $\alpha>1>\frac{d}{p}$ and $\alpha \geq \frac{\sigma_{1}}{2}+\frac{d}{4}$ for small enough $\varepsilon>0$, it is shown that

$$
\frac{\sigma_{1}+\sigma}{2} \leq \frac{\sigma_{1}}{2}+\frac{1}{2}+\frac{d}{4}<\frac{\sigma_{1}}{2}+\frac{d}{2 p}+\alpha<2 \alpha, \quad \frac{\sigma_{1}+\sigma}{2} \leq \frac{\sigma_{1}+1-\sigma_{0}}{2}+\alpha,
$$


and

$$
\frac{\sigma_{1}}{2}+\frac{d}{2 p}+\alpha>\frac{1}{2}-\frac{d}{4}+\frac{d}{2 p}+\alpha>1, \quad \frac{\sigma_{1}+1-\sigma_{0}}{2}+\alpha>1,
$$

since $p \leq d^{*}$ implies that $d / p \geq d / 2-1$. Therefore, we deduce (thanks to (4.2)) that

$$
\int_{0}^{t}\langle t-\tau\rangle^{-\frac{\sigma_{1}+\sigma}{2}}\left\|u \cdot \nabla a^{h}\right\|_{\dot{B}_{2, \infty}^{-\sigma_{1}}}^{\ell} d \tau \lesssim\langle t\rangle^{-\frac{\sigma_{1}+\sigma}{2}} \mathcal{D}_{p}^{2}(t) .
$$

To bound the term with $k(a) \nabla a^{h}$, we see that, using the composition inequality in Lebesgue spaces and the embeddings $\dot{B}_{2,1}^{\frac{d}{p}} \hookrightarrow \dot{B}_{p, 1}^{\sigma_{0}} \hookrightarrow L^{p^{*}}$, we get

$$
\|k(a)\|_{L^{p^{*}}} \lesssim\|a\|_{L^{p^{*}}} \lesssim\left\|a^{\ell}\right\|_{\dot{B}_{2,1}^{\frac{d}{p}}}+\left\|a^{h}\right\|_{\dot{B}_{p, 1}^{\sigma_{0}}} \lesssim\left\|a^{\ell}\right\|_{\dot{B}_{2,1}^{\frac{d}{p}}}+\left\|a^{h}\right\|_{\dot{B}_{p, 1}^{\frac{d}{p}}}
$$

Hence, taking advantage of (4.26) and Proposition 3.4,

$$
\left\|k(a) \nabla a^{h}\right\|_{\dot{B}_{2, \infty}^{-\sigma_{1}}}^{\ell} \lesssim\left(\|a\|_{\dot{B}_{p, 1}^{1-\frac{d}{p}}}+\left\|a^{\ell}\right\|_{\dot{B}_{2,1}^{\frac{d}{p}}}+\left\|a^{h}\right\|_{\dot{B}_{p, 1}^{\frac{d}{p}}}\right)\left\|\nabla a^{h}\right\|_{\dot{B}_{p, 1}^{\frac{d}{p}-1}}
$$

and one can thus bound the term corresponding to $k(a) \nabla a^{h}$ as $u \cdot \nabla a^{h}$. To bound the term with $a \operatorname{div} u^{h}$, we use (4.27), and get if $t \geq 2$,

$$
\begin{aligned}
& \int_{0}^{t}\langle t-\tau\rangle^{-\frac{\sigma_{1}+\sigma}{2}}\left\|a \operatorname{div} u^{h}\right\|_{\dot{B}_{2, \infty}^{-\sigma_{1}}}^{\ell} d \tau \\
\lesssim & \int_{0}^{t}\langle t-\tau\rangle^{-\frac{\sigma_{1}+\sigma}{2}}\left(\left\|a^{\ell}\right\|_{\dot{B}_{2,1}^{\frac{d}{p}}}+\|a\|_{\dot{B}_{p, 1}^{1-\frac{d}{p}}}\right)\left\|\operatorname{div} u^{h}\right\|_{\dot{B}_{p, 1}^{\frac{d}{p}-1}} d \tau \\
= & \left(\int_{0}^{1}+\int_{1}^{t}\right)(\cdots) d \tau \triangleq \widetilde{I}_{1}+\widetilde{I}_{2} .
\end{aligned}
$$

Remembering the definitions of $\mathcal{E}_{p}(t)$ and $\mathcal{D}_{p}(t)$ and using (4.28) and (4.29), we obtain

$$
\begin{aligned}
\widetilde{I}_{1} & \lesssim\langle t\rangle^{-\frac{\sigma_{1}+\sigma}{2}}\left(\sup _{0 \leq \tau \leq 1}\left\|a^{\ell}\right\|_{\dot{B}_{2,1}^{\frac{d}{p}}}+\sup _{0 \leq \tau \leq 1}\|a\|_{\dot{B}_{p, 1}^{1-\frac{d}{p}}}\right) \int_{0}^{1}\|u\|_{\dot{B}_{p, 1}^{\frac{d}{p}}}^{h} d \tau \\
& \lesssim\langle t\rangle^{-\frac{\sigma_{1}+\sigma}{2}} \mathcal{D}_{p}(1) \mathcal{E}_{p}(1),
\end{aligned}
$$

Using (4.2) and the fact that $\langle\tau\rangle \approx \tau$ when $\tau \geq 1$, one has for all $-\sigma_{1}<\sigma \leq \frac{d}{2}+1$,

$$
\begin{aligned}
\widetilde{I}_{2} \lesssim & \left(\sup _{1 \leq \tau \leq t}\langle\tau\rangle^{\frac{\sigma_{1}}{2}+\frac{d}{2 p}}\left\|a^{\ell}\right\|_{\dot{B}_{2,1}^{\frac{d}{p}}}+\sup _{1 \leq \tau \leq t}\langle\tau\rangle^{\frac{\sigma_{1}+1-\sigma_{0}}{2}}\|a\|_{\dot{B}_{p, 1}^{1-\frac{d}{p}}}\right)\left(\sup _{1 \leq \tau \leq t} \tau^{\alpha}\left\|u^{h}\right\|_{\dot{B}_{p, 1}^{\frac{d}{p}}}\right) \\
& \times \int_{1}^{t}\langle t-\tau\rangle^{-\frac{\sigma_{1}+\sigma}{2}}\left(\langle\tau\rangle^{-\frac{\sigma_{1}}{2}-\frac{d}{2 p}}+\langle\tau\rangle^{-\frac{\sigma_{1}+1-\sigma_{0}}{2}}\right)\langle\tau\rangle^{-\alpha} d \tau \lesssim\langle t\rangle^{-\frac{\sigma_{1}+\sigma}{2}} \mathcal{D}_{p}^{2}(t) .
\end{aligned}
$$

Therefore, we arrive at

$$
\int_{0}^{t}\langle t-\tau\rangle^{-\frac{\sigma_{1}+\sigma}{2}}\left\|a \operatorname{div} u^{h}\right\|_{\dot{B}_{2, \infty}^{-\sigma_{1}}}^{\ell} d \tau \lesssim\langle t\rangle^{-\frac{\sigma_{1}+\sigma}{2}}\left(\mathcal{D}_{p}(t) \mathcal{E}_{p}(t)+\mathcal{D}_{p}^{2}(t)\right) \text { for all } t \geq 2 .
$$

Owing to $\frac{d}{p}-1<1-\frac{d}{p}$ and $\frac{d}{p} \geq \frac{d}{2}-1$, we discover that

$$
\left\|(a, u)^{\ell}\right\|_{\dot{B}_{2,1}^{\frac{d}{p}}} \lesssim\|(a, u)\|_{\dot{B}_{2,1}^{\frac{d}{2}-1}}^{\ell}, \quad\left\|(a, u)^{\ell}\right\|_{\dot{B}_{p, 1}^{1-\frac{d}{p}}} \lesssim\left\|(a, u)^{\ell}\right\|_{\dot{B}_{p, 1}^{\frac{d}{p}-1}} \lesssim\|(a, u)\|_{\dot{B}_{2,1}^{\frac{d}{2}-1}}^{\ell}
$$


In the case $t \leq 2$, we have $\langle t\rangle \approx 1$ and $\langle t-\tau\rangle \approx 1$ for $0 \leq \tau \leq t \leq 2$, and

$$
\begin{aligned}
\int_{0}^{t}\langle t-\tau\rangle^{-\frac{\sigma_{1}+\sigma}{2}}\left\|a \operatorname{div} u^{h}\right\|_{\dot{B}_{2, \infty}^{-\sigma_{1}}}^{\ell} d \tau & \lesssim\left(\|a\|_{L_{t}^{\infty}\left(\dot{B}_{2,1}^{\frac{d}{2}-1}\right)}^{\ell}+\|a\|_{L_{t}^{\infty}\left(\dot{B}_{p, 1}^{\frac{d}{p}}\right)}^{h}\right)\|u\|_{L_{t}^{1}\left(\dot{B}_{p, 1}^{\frac{d}{p}}\right)}^{h} \\
& \lesssim \mathcal{E}_{p}^{2}(t) .
\end{aligned}
$$

In order to bound the term with $g_{3}\left(a, u^{h}\right)$, we mimic the proof of $(4.31)$ and get

$$
\left\|g_{3}\left(a, u^{h}\right)\right\|_{\dot{B}_{2, \infty}^{-\sigma_{1}}}^{\ell} \lesssim\left(\|a\|_{\dot{B}_{p, 1}^{1-\frac{d}{p}}}+\left\|a^{\ell}\right\|_{\dot{B}_{2,1}^{\frac{d}{p}}}+\left\|a^{h}\right\|_{\dot{B}_{p, 1}^{\frac{d}{p}}}\right)\left\|\nabla^{2} u^{h}\right\|_{\dot{B}_{p, 1}^{\frac{d}{p}-1}},
$$

and one can conclude that exactly as the previous term $a \operatorname{div} u^{h}$ that

$$
\int_{0}^{t}\langle t-\tau\rangle^{-\frac{\sigma_{1}+\sigma}{2}}\left\|g_{3}\left(a, u^{h}\right)\right\|_{\dot{B}_{2, \infty}^{-\sigma_{1}}}^{\ell} d \tau \lesssim\langle t\rangle^{-\frac{\sigma_{1}+\sigma}{2}}\left(\mathcal{D}_{p}(t) \mathcal{E}_{p}(t)+\mathcal{D}_{p}^{2}(t)+\mathcal{E}_{p}^{2}(t)\right) .
$$

To handle the term with $u \cdot \nabla u^{h}$, we note that, thanks to (4.27) and interpolation,

$$
\left\|u \cdot \nabla u^{h}\right\|_{\dot{B}_{2, \infty}^{-\sigma_{1}}}^{\ell} \lesssim\left(\left\|u^{\ell}\right\|_{\dot{B}_{2,1}^{\frac{d}{p}}}+\left\|u^{\ell}\right\|_{\dot{B}_{p, 1}^{1-\frac{d}{p}}}+\left\|u^{h}\right\|_{\dot{B}_{p, 1}^{\frac{d}{p}-1}}\right)\left\|\nabla u^{h}\right\|_{\dot{B}_{p, 1}^{\frac{d}{p}}} .
$$

If $t \geq 2$, then we write that

$$
\begin{aligned}
& \int_{0}^{t}\langle t-\tau\rangle^{-\frac{\sigma_{1}+\sigma}{2}}\left\|u \cdot \nabla u^{h}\right\|_{\dot{B}_{2, \infty}^{-\sigma_{1}}}^{\ell} d \tau \\
\lesssim & \int_{0}^{t}\langle t-\tau\rangle^{-\frac{\sigma_{1}+\sigma}{2}}\left(\left\|u^{\ell}\right\|_{\dot{B}_{2,1}^{\frac{d}{p}}}+\left\|u^{\ell}\right\|_{\dot{B}_{p, 1}^{1-\frac{d}{p}}}+\left\|u^{h}\right\|_{\dot{B}_{p, 1}^{\frac{d}{p}-1}}\right)\left\|\nabla u^{h}\right\|_{\dot{B}_{p, 1}^{\frac{d}{p}}} d \tau \\
= & \left(\int_{0}^{1}+\int_{1}^{t}\right)(\cdots) d \tau \triangleq \widetilde{J}_{1}+\widetilde{J}_{2} .
\end{aligned}
$$

It just follows from $(4.28),(4.29)$ and the definitions of $\mathcal{D}_{p}(t)$ and $\mathcal{E}_{p}(t)$ that

$$
\widetilde{J}_{1} \lesssim\langle t\rangle^{-\frac{\sigma_{1}+\sigma}{2}}\left(\mathcal{D}_{p}(1) \mathcal{E}_{p}(1)+\mathcal{E}_{p}^{2}(1)\right) .
$$

and also that

$$
\begin{aligned}
\widetilde{J}_{2} & \lesssim \mathcal{D}_{p}^{2}(t) \int_{1}^{t}\langle t-\tau\rangle^{-\frac{\sigma_{1}+\sigma}{2}}\left(\langle\tau\rangle^{-\frac{\sigma_{1}}{2}-\frac{d}{2 p}}+\langle\tau\rangle^{-\frac{\sigma_{1}+1-\sigma_{0}}{2}}+\langle\tau\rangle^{-\alpha}\right)\langle\tau\rangle^{-\alpha} d \tau \\
& \lesssim\langle t\rangle^{-\frac{\sigma_{1}+\sigma}{2}} \mathcal{D}_{p}^{2}(t) .
\end{aligned}
$$

Consequently, for all $t \geq 2$, we have

$$
\int_{0}^{t}\langle t-\tau\rangle^{-\frac{\sigma_{1}+\sigma}{2}}\left\|u \cdot \nabla u^{h}\right\|_{\dot{B}_{2, \infty}^{-\sigma_{1}}}^{\ell} d \tau \lesssim\langle t\rangle^{-\frac{\sigma_{1}+\sigma}{2}}\left(\mathcal{D}_{p}(t) \mathcal{E}_{p}(t)+\mathcal{D}_{p}^{2}(t)+\mathcal{E}_{p}^{2}(t)\right) .
$$

In the case $t \leq 2$, we have $\langle t\rangle \approx 1$ and $\langle t-\tau\rangle \approx 1$ for $0 \leq \tau \leq t \leq 2$. Furthermore, it follows from (4.32) that

$$
\begin{aligned}
\int_{0}^{t}\langle t-\tau\rangle^{-\frac{\sigma_{1}+\sigma}{2}}\left\|u \cdot \nabla u^{h}\right\|_{\dot{B}_{2, \infty}^{-\sigma_{1}}}^{\ell} d \tau & \lesssim\left(\|u\|_{L_{t}^{\infty}\left(\dot{B}_{2,1}^{\frac{d}{2}-1}\right)}^{\ell}+\|u\|_{L_{t}^{\infty}\left(\dot{B}_{p, 1}^{\frac{d}{p}-1}\right)}^{h}\|u\|_{L_{t}^{1}\left(\dot{B}_{p, 1}^{\frac{d}{p}+1}\right)}^{h}\right. \\
& \lesssim \mathcal{E}_{p}^{2}(t) .
\end{aligned}
$$


Lastly, we consider the term with $g_{4}\left(a, u^{h}\right)$. Applying (3.6) with $s=1-\frac{d}{p}$ yields for any smooth function $K$ vanishing at 0 ,

$$
\left\|\nabla K(a) \otimes \nabla u^{h}\right\|_{\dot{B}_{2, \infty}^{-\sigma_{0}}}^{\ell} \lesssim\left(\left\|\nabla u^{h}\right\|_{\dot{B}_{p, 1}^{1-\frac{d}{p}}}+\sum_{j=j_{0}}^{j_{0}+N_{0}-1}\left\|\dot{\Delta}_{j} \nabla u^{h}\right\|_{L^{p^{*}}}\right)\|\nabla K(a)\|_{\dot{B}_{p, 1}^{\frac{d}{p}-1}} .
$$

As $p^{*} \geq p$, we get from Bernstein inequality that for $j_{0} \leq j<j_{0}+N_{0}$,

$$
\left\|\dot{\Delta}_{j} \nabla u^{h}\right\|_{L^{p^{*}}} \lesssim\left\|\dot{\Delta}_{j} \nabla u^{h}\right\|_{L^{p}}
$$

Hence, using Proposition 3.4, the fact that $\sigma_{1} \leq \sigma_{0}$ and that $1-\frac{d}{p}<\frac{d}{p}$, we have

$$
\left\|g_{4}\left(a, u^{h}\right)\right\|_{\dot{B}_{2, \infty}^{-\sigma_{1}}}^{\ell} \lesssim\left\|g_{4}\left(a, u^{h}\right)\right\|_{\dot{B}_{2, \infty}^{-\sigma_{0}}}^{\ell} \lesssim\|a\|_{\dot{B}_{p, 1}^{\frac{d}{p}}}\left\|\nabla u^{h}\right\|_{\dot{B}_{p, 1}^{1-\frac{d}{p}}} \lesssim\|a\|_{\dot{B}_{p, 1}^{\frac{d}{p}}}\left\|\nabla u^{h}\right\|_{\dot{B}_{p, 1}^{\frac{d}{p}}}
$$

Therefore,

$$
\begin{aligned}
\int_{0}^{t}\langle t-\tau\rangle^{-\frac{\sigma_{1}+\sigma}{2}}\left\|g_{4}\left(a, u^{h}\right)\right\|_{\dot{B}_{2, \infty}^{-\sigma_{1}}}^{\ell} d \tau & \lesssim \int_{0}^{t}\langle t-\tau\rangle^{-\frac{\sigma_{1}+\sigma}{2}}\|a\|_{\dot{B}_{p, 1}^{\frac{d}{p}}}\left\|\nabla u^{h}\right\|_{\dot{B}_{p, 1}^{\frac{d}{p}}} d \tau \\
& =\left(\int_{0}^{1}+\int_{1}^{t}\right)(\cdots) d \tau \triangleq \widetilde{K}_{1}+\widetilde{K}_{2} .
\end{aligned}
$$

For $\widetilde{K}_{1}$, it is clear that

$$
\widetilde{K}_{1} \lesssim\langle t\rangle^{-\frac{\sigma_{1}+\sigma}{2}} \mathcal{E}_{p}^{2}(1)
$$

and that, owing to the definition of $\mathcal{D}_{p}(t)$ and (4.2), (4.10), if $t \geq 2$,

$$
\begin{aligned}
\widetilde{K}_{2} \lesssim & \left(\sup _{1 \leq \tau \leq t}\langle\tau\rangle^{\frac{\sigma_{1}}{2}+\frac{d}{4}}\|a\|_{\dot{B}_{p, 1}^{\frac{d}{p}}}\right)\left(\sup _{1 \leq \tau \leq t} \tau^{\alpha}\left\|\nabla u^{h}\right\|_{\dot{B}_{p, 1}^{\frac{d}{p}}}\right) \\
& \times \int_{1}^{t}\langle t-\tau\rangle^{-\frac{\sigma_{1}+\sigma}{2}}\langle\tau\rangle^{-\frac{\sigma_{1}}{2}-\frac{d}{4}-\alpha} d \tau \lesssim\langle t\rangle^{-\frac{\sigma_{1}+\sigma}{2}} \mathcal{D}_{p}^{2}(t) .
\end{aligned}
$$

Hence, the proof of Lemma 4.5 is complete.

Combining those estimates in Lemmas 4.2-4.5, we achieve the inequality (4.6). Consequently, the proof of Proposition 4.1 is finished. Furthermore, together with (4.5) for bounding the term of (4.4) pertaining to the data, we deduce that

$$
\langle t\rangle^{\frac{\sigma_{1}+\sigma}{2}}\|(a, u)(t)\|_{\dot{B}_{2,1}^{\sigma}}^{\ell} \lesssim \mathcal{D}_{p, 0}+\mathcal{D}_{p}^{2}(t)+\mathcal{E}_{p}^{2}(t) \text { for all } t \geq 0,
$$

provided that $-\sigma_{1}<\sigma \leq \frac{d}{2}+1$.

4.2. Second step: Decay estimates for the high frequencies of $(\nabla a, u)$. This section is devoted to bound the second term of $\mathcal{D}_{p}(t)$, which follows from the elementary energy approach of $L^{p}$ type. For the end, we first present a time-weighted product estimate, which can be regarded as the generalization of Proposition 3.2.

Lemma 4.6. Let $s>0$ and $1 \leq p, r \leq \infty$. For $T>0$ and $\beta=\beta_{1}+\beta_{2}$ with $\beta_{1}, \beta_{2} \geq 0$, one has

$$
\left\|t^{\beta} u v\right\|_{\widetilde{L}_{T}^{\theta}\left(\dot{B}_{p, r}^{s}\right)} \lesssim\left\|t^{\beta_{1}} u\right\|_{L_{T}^{\theta_{1}}\left(L^{\infty}\right)}\left\|t^{\beta_{2}} v\right\|_{\widetilde{L}_{T}^{\theta_{2}\left(\dot{B}_{p, r}^{s}\right)}}+\left\|t^{\beta_{1}} v\right\|_{L_{T}^{\theta_{1}}\left(L^{\infty}\right)}\left\|t^{\beta_{2}} u\right\|_{\widetilde{L}_{T}^{\theta_{2}}\left(\dot{B}_{p, r}^{s}\right)},
$$

where $1 / \theta=1 / \theta_{1}+1 / \theta_{2}$. 
Proof. In terms of Bony's decomposition (see the definition in Chap. 2 of [1]), we have

$$
t^{\beta} u v=\mathcal{T}_{t^{\beta_{1}} u}\left(t^{\beta_{2}} v\right)+\mathcal{T}_{t^{\beta_{1}}}\left(t^{\beta_{2}} u\right)+\mathcal{R}\left(t^{\beta_{1}} u, t^{\beta_{2}} v\right) .
$$

According to those results of continuity for the paraproduct and the remainder (see $[1])$, it is not difficult to get

$$
\begin{aligned}
& \left\|\mathcal{T}_{t^{\beta_{1}} u}\left(t^{\beta_{2}} v\right)\right\|_{\widetilde{L}_{T}^{\theta}\left(\dot{B}_{p, r}^{s}\right)} \lesssim\left\|t^{\beta_{1}} u\right\|_{L_{T}^{\theta_{1}}\left(L^{\infty}\right)}\left\|t^{\beta_{2}} v\right\|_{\widetilde{L}_{T}^{\theta_{2}}\left(\dot{B}_{p, r}^{s}\right)}, \\
& \left\|\mathcal{T}_{t^{\beta_{1}}}\left(t^{\beta_{2}} u\right)\right\|_{\widetilde{L}_{T}^{\theta}\left(\dot{B}_{p, r}^{s}\right)} \lesssim\left\|t^{\beta_{1}} v\right\|_{L_{T}^{\theta_{1}}\left(L^{\infty}\right)}\left\|t^{\beta_{2}} u\right\|_{\widetilde{L}_{T}^{\theta_{2}}\left(\dot{B}_{p, r}^{s}\right)}, \\
& \left\|\mathcal{R}\left(t^{\beta_{1}} u, t^{\beta_{2}} v\right)\right\|_{\tilde{L}_{T}^{\theta}\left(\dot{B}_{p, r}^{s}\right)} \lesssim\left\|t^{\beta_{1}} u\right\|_{\widetilde{L}_{T}^{\theta_{1}}\left(\dot{B}_{\infty, \infty}^{0}\right)}\left\|t^{\beta_{2}} v\right\|_{\widetilde{L}_{T}^{\theta_{2}}\left(\dot{B}_{p, r}^{s}\right)} .
\end{aligned}
$$

Hence, Remark 3.1 and the embedding $L^{\infty} \hookrightarrow \dot{B}_{\infty, \infty}^{0}$ leads to the desired conclusion.

Proposition 4.2. If $p$ satisfies (2.2), then it holds that for all $T \geq 0$,

$$
\left\|\langle t\rangle^{\alpha}(\nabla a, u)\right\|_{\widetilde{L}_{T}^{\infty}\left(\dot{B}_{p, 1}^{\frac{d}{p}-1}\right)}^{h} \lesssim\left\|\left(\nabla a_{0}, u_{0}\right)\right\|_{\dot{B}_{p, 1}^{\frac{d}{p}-1}}^{h}+\mathcal{E}_{p}^{2}(T)+\mathcal{D}_{p}^{2}(T)
$$

with $\alpha=\sigma_{1}+\frac{d}{2}+\frac{1}{2}-\varepsilon$ for sufficiently small $\varepsilon>0$, where $\mathcal{E}_{p}(T)$ and $\mathcal{D}_{p}(T)$ are defined by (4.1) and (2.6), respectively.

Proof. The proof of Proposition 4.2 is to exhibit the optimal decay exponents for the high frequencies of $(a, u)$ with the assumption of (2.3)-(2.4). Let $\mathcal{P} \triangleq \operatorname{Id}+\nabla(-\Delta)^{-1} \operatorname{div}$ be the Leray projector onto divergence-free vector-fields. Then $\mathcal{P} u$ satisfies a heat equation

$$
\partial_{t} \mathcal{P} u-\mu_{\infty} \Delta \mathcal{P} u=\mathcal{P} g .
$$

Let us introduce the effective velocity $w$ (see (2.7)) as in [15], which was originated from Hoff's viscous effective flux in [16]. It is easy to check that $w$ fulfills some constant coefficient heat equation, while $a$ satisfies a damped transport equation. Precisely, one has

$$
\left\{\begin{array}{l}
\partial_{t} a+a=f-\operatorname{div} w \\
\partial_{t} w-\Delta w=\nabla(-\Delta)^{-1}(f-\operatorname{div} g)+w-(-\Delta)^{-1} \nabla a .
\end{array}\right.
$$

Then, by performing the $L^{p}$ energy method in [10], we can end up with

$$
\begin{aligned}
\left\|\langle t\rangle^{\alpha}(\nabla a, u)\right\|_{\widetilde{L}_{T}^{\infty}\left(\dot{B}_{p, 1}^{\frac{d}{p}-1}\right)}^{h} \lesssim & \left\|\left(\nabla a_{0}, u_{0}\right)\right\|_{\dot{B}_{p, 1}^{\frac{d}{p}-1}}^{h} \\
& +\sum_{j \geq j_{0}-1} \sup _{0 \leq t \leq T}\left(\langle t\rangle^{\alpha} \int_{0}^{t} e^{-c_{0}(t-\tau)} 2^{j\left(\frac{d}{p}-1\right)} Z_{j}(\tau) d \tau\right) .
\end{aligned}
$$

with $Z_{j} \triangleq Z_{j}^{1}+\cdots+Z_{j}^{5}$ and

$$
\begin{array}{ll}
Z_{j}^{1} \triangleq\left\|\dot{\Delta}_{j}(a u)\right\|_{L^{p}}, & Z_{j}^{2} \triangleq\left\|g_{j}\right\|_{L^{p}}, \quad Z_{j}^{3} \triangleq\left\|\nabla \dot{\Delta}_{j}(a \operatorname{div} u)\right\|_{L^{p}}, \\
Z_{j}^{4} \triangleq\left\|R_{j}\right\|_{L^{p}}, & Z_{j}^{5} \triangleq\|\operatorname{div} u\|_{L^{\infty}}\left\|\nabla a_{j}\right\|_{L^{p}},
\end{array}
$$

where $a_{j} \triangleq \dot{\Delta}_{j} a, g_{j} \triangleq \dot{\Delta}_{j} g$ and $R_{j} \triangleq\left[u \cdot \nabla, \nabla \dot{\Delta}_{j}\right] a$.

Firstly, we observe that

$$
\sum_{j \geq j_{0}-1} \sup _{0 \leq t \leq 2}\left(\langle t\rangle^{\alpha} \int_{0}^{t} e^{-c_{0}(t-\tau)} 2^{j\left(\frac{d}{p}-1\right)} Z_{j}(\tau) d \tau\right) \lesssim \int_{0}^{2} \sum_{j \geq j_{0}-1} 2^{j\left(\frac{d}{p}-1\right)} Z_{j}(\tau) d \tau .
$$


Furthermore, it follows from Propositions 3.2 and 3.5 that

$$
\int_{0}^{2} \sum_{j \geq j_{0}-1} 2^{j\left(\frac{d}{p}-1\right)} Z_{j}(\tau) d \tau \lesssim \int_{0}^{2}\left(\|(a u, g)\|_{\dot{B}_{p, 1}^{\frac{d}{p}-1}}^{h}+\|\nabla u\|_{\dot{B}_{p, 1}^{\frac{d}{p}}}\|a\|_{\dot{B}_{p, 1}^{\frac{d}{p}}}\right) d \tau .
$$

It is obvious that the last term of the r.h.s. of (4.36) may be bounded by $C \mathcal{E}_{p}^{2}(2)$ and thanks to Proposition 3.2, we arrive at

$$
\|a u\|_{L_{t}^{1}\left(\dot{B}_{p, 1}^{\frac{d}{p}-1}\right)}^{h} \lesssim\|a u\|_{L_{t}^{1}\left(\dot{B}_{p, 1}^{\frac{d}{p}}\right)} \lesssim\|a\|_{L_{t}^{2}\left(\dot{B}_{p, 1}^{\frac{d}{p}}\right)}\|u\|_{L_{t}^{2}\left(\dot{B}_{p, 1}^{\frac{d}{p}}\right)} .
$$

It follows from Propositions 3.2 and 3.4 that

$$
\begin{aligned}
\|g\|_{L_{t}^{1}\left(\dot{B}_{p, 1}^{\frac{d}{p}-1}\right)}^{h} & \left(\|u\|_{L_{t}^{\infty}\left(\dot{B}_{p, 1}^{\frac{d}{p}-1}\right)}\|\nabla u\|_{L_{t}^{1}\left(\dot{B}_{p, 1}^{\frac{d}{p}}\right)}+\|a\|_{L_{t}^{\infty}\left(\dot{B}_{p, 1}^{\frac{d}{p}}\right)}\|\nabla u\|_{L_{t}^{1}\left(\dot{B}_{p, 1}^{\frac{d}{p}}\right)}\right. \\
& \left.+\|a\|_{L_{t}^{2}\left(\dot{B}_{p, 1}^{\frac{d}{p}}\right)}\|\nabla a\|_{L_{t}^{2}\left(\dot{B}_{p, 1}^{\frac{d}{p}-1}\right)}\right) .
\end{aligned}
$$

Combining interpolation and the definition of $\mathcal{E}_{p}(t)$, we deduce that

$$
\|a\|_{L_{t}^{2}\left(\dot{B}_{p, 1}^{\frac{d}{p}}\right)}+\|u\|_{L_{t}^{2}\left(\dot{B}_{p, 1}^{\frac{d}{p}}\right)} \lesssim \mathcal{E}_{p}(t) .
$$

Furthermore, we can conclude that the first term in the right-hand side of (4.36) may be bounded by $\mathcal{E}_{p}^{2}(2)$. We end up with

$$
\sum_{j \geq j_{0}-1} \sup _{0 \leq t \leq 2}\left(\langle t\rangle^{\alpha} \int_{0}^{t} e^{-c_{0}(t-\tau)} 2^{j\left(\frac{d}{p}-1\right)} Z_{j}(\tau) d \tau\right) \lesssim \mathcal{E}_{p}^{2}(2)
$$

Secondly, let us handle the supremum for $2 \leq t \leq T$ in the last term of (4.35). To do this, one can split the integral on $[0, t]$ into integrals $[0,1]$ and $[1, t]$. The $[0,1]$ part of the integral may be bounded by $\mathcal{E}_{p}^{2}(1)$. Indeed, owing to $e^{-c_{0}(t-\tau)} \leq e^{-\frac{c_{0} t}{2}}$ for $2 \leq t \leq T$ and $0 \leq \tau \leq 1$, we obtain

$$
\begin{aligned}
& \sum_{j \geq j_{0}-1} \sup _{2 \leq t \leq T}\left(\langle t\rangle^{\alpha} \int_{0}^{1} e^{-c_{0}(t-\tau)} 2^{j\left(\frac{d}{p}-1\right)} Z_{j}(\tau) d \tau\right) \\
\lesssim & \sum_{j \geq j_{0}-1} \sup _{2 \leq t \leq T}\left(\langle t\rangle^{\alpha} e^{-\frac{c_{0}}{2} t} \int_{0}^{1} 2^{j\left(\frac{d}{p}-1\right)} Z_{j}(\tau) d \tau\right) \lesssim \int_{0}^{1} \sum_{j \geq j_{0}-1} 2^{j\left(\frac{d}{p}-1\right)} Z_{j}(\tau) d \tau .
\end{aligned}
$$

Hence, following from the procedure leading to (4.37), we arrive at

$$
\sum_{j \geq j_{0}-1} \sup _{2 \leq t \leq T}\left(\langle t\rangle^{\alpha} \int_{0}^{1} e^{-c_{0}(t-\tau)} 2^{j\left(\frac{d}{p}-1\right)} Z_{j}(\tau) d \tau\right) \lesssim \mathcal{E}_{p}^{2}(1) .
$$

In order to handle the $[1, t]$ part of the integral for $2 \leq t \leq T$, we start from

$$
\sum_{j \geq j_{0}-1} \sup _{2 \leq t \leq T}\left(\langle t\rangle^{\alpha} \int_{1}^{t} e^{-c_{0}(t-\tau)} 2^{j\left(\frac{d}{p}-1\right)} Z_{j}(\tau) d \tau\right) \lesssim \sum_{j \geq j_{0}-1} 2^{j\left(\frac{d}{p}-1\right)} \sup _{1 \leq t \leq T} t^{\alpha} Z_{j}(t) .
$$

To bound the contribution of $Z_{j}^{1}$ and $Z_{j}^{2}$ in (4.35), we use the fact that

$$
\sum_{j \geq j_{0}-1} 2^{j\left(\frac{d}{p}-1\right)} \sup _{1 \leq t \leq T} t^{\alpha}\left(Z_{j}^{1}(t)+Z_{j}^{2}(t)\right) \lesssim\left\|t^{\alpha}(a u, g)\right\|_{\widetilde{L}_{T}^{\infty}\left(\dot{B}_{p, 1}^{\frac{d}{p}-1}\right)}^{h} .
$$


For the term au, we decompose it as follows

$$
a u=a u^{h}+a^{h} u^{\ell}+a^{\ell} u^{\ell} .
$$

Product laws in Proposition 3.2 adapted to tilde spaces ensure that

$$
\begin{aligned}
\left\|t^{\alpha} a u^{h}\right\|_{\widetilde{L}_{T}^{\infty}\left(\dot{B}_{p, 1}^{\frac{d}{p}-1}\right)}^{h} & \lesssim\|a\|_{\widetilde{L}_{T}^{\infty}\left(\dot{B}_{p, 1}^{\frac{d}{p}}\right)}\left\|t^{\alpha} u^{h}\right\|_{\widetilde{L}_{T}^{\infty}\left(\dot{B}_{p, 1}^{\frac{d}{p}-1}\right)} \lesssim \mathcal{E}_{p}(T) \mathcal{D}_{p}(T), \\
\left\|t^{\alpha} a^{h} u^{\ell}\right\|_{\widetilde{L}_{T}^{\infty}\left(\dot{B}_{p, 1}^{\frac{d}{p}-1}\right)}^{h} & \lesssim\left\|t^{\alpha} a^{h}\right\|_{\widetilde{L}_{T}^{\infty}\left(\dot{B}_{p, 1}^{\frac{d}{p}}\right)}\left\|u^{\ell}\right\|_{\widetilde{L}_{T}^{\infty}\left(\dot{B}_{p, 1}^{\frac{d}{p}-1}\right)} \lesssim \mathcal{D}_{p}(T) \mathcal{E}_{p}(T) .
\end{aligned}
$$

Bernstein inequality and embedding imply that

$$
\left\|t^{\alpha} a^{\ell} u^{\ell}\right\|_{\widetilde{L}_{T}^{\infty}\left(\dot{B}_{p, 1}^{\frac{d}{p}-1}\right)}^{h} \lesssim\left\|t^{\alpha} a^{\ell} u^{\ell}\right\|_{\widetilde{L}_{T}^{\infty}\left(\dot{B}_{2,1}^{\frac{d}{2}+1}\right)},
$$

and thus it follows from Lemma 4.6 (noticing that $\dot{B}_{2,1}^{\frac{d}{2}} \hookrightarrow L^{\infty}$ ) that

$$
\begin{aligned}
\left\|t^{\alpha} a^{\ell} u^{\ell}\right\|_{\widetilde{L}_{T}^{\infty}\left(\dot{B}_{p, 1}^{\frac{d}{p}-1}\right)}^{h} \lesssim & \left\|t^{\frac{\sigma_{1}}{2}+\frac{d}{4}} a^{\ell}\right\|_{L_{T}^{\infty}\left(\dot{B}_{2,1}^{\frac{d}{2}}\right)}\left\|t^{\frac{\sigma_{1}}{2}+\frac{d}{4}+\frac{1}{2}-\varepsilon} u^{\ell}\right\|_{\widetilde{L}_{T}^{\infty}\left(\dot{B}_{2,1}^{\frac{d}{2}+1}\right)} \\
& +\left\|t^{\frac{\sigma_{1}}{2}+\frac{d}{4}} u^{\ell}\right\|_{L_{T}^{\infty}\left(\dot{B}_{2,1}^{\frac{d}{2}}\right)}\left\|t^{\frac{\sigma_{1}}{2}+\frac{d}{4}+\frac{1}{2}-\varepsilon} a^{\ell}\right\|_{\widetilde{L}_{T}^{\infty}\left(\dot{B}_{2,1}^{\frac{d}{2}+1}\right)} .
\end{aligned}
$$

Owing to the definition of $\mathcal{D}_{p}(t)$ and Remark 3.1, we have

$$
\left\|t^{\frac{\sigma_{1}}{2}+\frac{d}{4}+\frac{1}{2}-\varepsilon}(a, u)^{\ell}(t)\right\|_{\widetilde{L}_{T}^{\infty}\left(\dot{B}_{2,1}^{\frac{d}{2}+1}\right)} \lesssim\left\|t^{\frac{\sigma_{1}}{2}+\frac{d}{4}+\frac{1}{2}-\varepsilon}(a, u)^{\ell}(t)\right\|_{L_{T}^{\infty}\left(\dot{B}_{2,1}^{\frac{d}{2}+1-2 \varepsilon}\right)} \lesssim \mathcal{D}_{p}(T),
$$

whence

$$
\left\|t^{\alpha} a^{\ell} u^{\ell}\right\|_{\tilde{L}_{T}^{\infty}\left(\dot{B}_{p, 1}^{\frac{d}{p}-1}\right)}^{h} \lesssim \mathcal{D}_{p}^{2}(T)
$$

In what follows, we shall use repeatedly the two inequalities for sufficiently small $\varepsilon>0$ :

$$
\begin{aligned}
& \left\|t^{\frac{\sigma_{1}}{2}+\frac{d}{4}+\frac{m}{2}-\frac{\varepsilon}{2}}\left(\nabla^{m} a^{\ell}, \nabla^{m} u^{\ell}\right)(\tau)\right\|_{\tilde{L}_{T}^{\infty}\left(\dot{B}_{p, 1}^{\frac{d}{p}}\right)} \lesssim \mathcal{D}_{p}(T), \\
& \left\|t^{\frac{\sigma_{1}}{2}+\frac{d}{4}+\frac{m}{2}-\frac{\varepsilon}{2}}\left(\nabla a^{h}, u^{h}\right)(\tau)\right\|_{\widetilde{L}_{T}^{\infty}\left(\dot{B}_{p, 1}^{\frac{d}{p}}-1\right)} \lesssim \mathcal{D}_{p}(T) \text { for } m=0,1 .
\end{aligned}
$$

Indeed, it follows from the embedding, the definition of $\mathcal{D}_{p}(t)$ and tilde norms that

$$
\begin{aligned}
\left\|t^{\frac{\sigma_{1}}{2}+\frac{d}{4}+\frac{m}{2}-\frac{\varepsilon}{2}}\left(\nabla^{m} a^{\ell}, \nabla^{m} u^{\ell}\right)\right\|_{\widetilde{L}_{T}^{\infty}\left(\dot{B}_{p, 1}^{\frac{d}{p}}\right)} & \lesssim\left\|t^{\frac{\sigma_{1}}{2}+\frac{d}{4}+\frac{m}{2}-\frac{\varepsilon}{2}}\left(\nabla^{m} a^{\ell}, \nabla^{m} u^{\ell}\right)\right\|_{\widetilde{L}_{T}^{\infty}\left(\dot{B}_{2,1}^{\frac{d}{2}}\right)} \\
& \lesssim\left\|\langle t\rangle^{\frac{\sigma_{1}}{2}+\frac{d}{4}+\frac{m}{2}-\frac{\varepsilon}{2}}(a, u)\right\|_{L_{T}^{\infty}\left(\dot{B}_{2,1}^{\frac{d}{2}+m-\varepsilon}\right)}^{\ell} \\
& \lesssim \mathcal{D}_{p}(T)
\end{aligned}
$$

and also that, due to $\alpha \geq \frac{\sigma_{1}}{2}+\frac{d}{4}+\frac{m}{2}-\frac{\varepsilon}{2}$ for small enough $\varepsilon>0$,

$$
\begin{aligned}
\left\|t^{\frac{\sigma_{1}}{2}+\frac{d}{4}+\frac{m}{2}-\frac{\varepsilon}{2}}\left(\nabla a^{h}, u^{h}\right)\right\|_{\widetilde{L}_{T}^{\infty}\left(\dot{B}_{p, 1}^{\frac{d}{p}-1}\right)} & \lesssim\left\|\langle t\rangle^{\alpha}\left(\nabla a^{h}, u^{h}\right)(\tau)\right\|_{\widetilde{L}_{T}^{\infty}\left(\dot{B}_{p, 1}^{\frac{d}{p}-1}\right)} \\
& \lesssim \mathcal{D}_{p}(T) \text { for } m=0,1 .
\end{aligned}
$$

Next, let us pay attention to (4.40) corresponding to the nonlinear term $g$, which consists of

$$
u \cdot \nabla u, \quad k(a) \nabla a, \quad g_{3}(a, u) \text { and } g_{4}(a, u) .
$$


Regarding the convection term $u \cdot \nabla u$, we write

$$
u \cdot \nabla u=u \cdot \nabla u^{h}+u^{h} \cdot \nabla u^{\ell}+u^{\ell} \cdot \nabla u^{\ell} .
$$

Then Proposition 3.2 adapted to tilde spaces and (4.41)-(4.42) ensure that

$$
\begin{aligned}
& \left\|t^{\alpha}\left(u \cdot \nabla u^{h}\right)\right\|_{\widetilde{L}_{T}^{\infty}\left(\dot{B}_{p, 1}^{\frac{d}{p}-1}\right)}^{h} \lesssim\|u\|_{\widetilde{L}_{T}^{\infty}\left(\dot{B}_{p, 1}^{\frac{d}{p}-1}\right)}\left\|t^{\alpha} \nabla u^{h}\right\|_{\widetilde{L}_{T}^{\infty}\left(\dot{B}_{p, 1}^{\frac{d}{p}}\right)} \lesssim \mathcal{E}_{p}(T) \mathcal{D}_{p}(T), \\
& \left\|t^{\alpha}\left(u^{h} \cdot \nabla u^{\ell}\right)\right\|_{\widetilde{L}_{T}^{\infty}\left(\dot{B}_{p, 1}^{\frac{d}{p}-1}\right)}^{h} \lesssim\left\|t^{\frac{\sigma_{1}}{2}+\frac{d}{4}-\frac{\varepsilon}{2}} u^{h}\right\|_{\widetilde{L}_{T}^{\infty}\left(\dot{B}_{p, 1}^{\frac{d}{p}-1}\right)}\left\|t^{\frac{\sigma_{1}}{2}+\frac{d}{4}+\frac{1}{2}-\frac{\varepsilon}{2}} \nabla u^{\ell}\right\|_{\widetilde{L}_{T}^{\infty}\left(\dot{B}_{p, 1}^{\frac{d}{p}}\right)} \\
& \lesssim \mathcal{D}_{p}^{2}(T)
\end{aligned}
$$

and

$$
\begin{aligned}
\left\|t^{\alpha}\left(u^{\ell} \cdot \nabla u^{\ell}\right)\right\|_{\tilde{L}_{T}^{\infty}\left(\dot{B}_{p, 1}^{\frac{d}{p}-1}\right)}^{h} & \lesssim\left\|t^{\alpha}\left(u^{\ell} \cdot \nabla u^{\ell}\right)\right\|_{\widetilde{L}_{T}^{\infty}\left(\dot{B}_{p, 1}^{\frac{d}{p}}\right)} \\
& \lesssim\left\|t^{\frac{\sigma_{1}}{2}+\frac{d}{4}-\frac{\varepsilon}{2}} u^{\ell}\right\|_{\widetilde{L}_{T}^{\infty}\left(\dot{B}_{p, 1}^{\frac{d}{p}}\right)}\left\|t^{\frac{\sigma_{1}}{2}+\frac{d}{4}+\frac{1}{2}-\frac{\varepsilon}{2}} \nabla u^{\ell}\right\|_{\widetilde{L}_{T}^{\infty}\left(\dot{B}_{p, 1}^{\frac{d}{p}}\right)} \\
& \lesssim \mathcal{D}_{p}^{2}(T) .
\end{aligned}
$$

To bound the term containing $k(a) \nabla a$, we use that

$$
\begin{aligned}
&\left\|t^{\alpha} k(a) \nabla a^{\ell}\right\|_{\widetilde{L}_{T}^{\infty}\left(\dot{B}_{p, 1}^{\frac{d}{p}-1}\right)}^{h} \lesssim\left\|t^{\frac{\sigma_{1}}{2}+\frac{d}{4}-\frac{\varepsilon}{2}} a\right\|_{\widetilde{L}_{T}^{\infty}\left(\dot{B}_{p, 1}^{\frac{d}{p}}\right)}\left\|t^{\frac{\sigma_{1}}{2}+\frac{d}{4}+\frac{1}{2}-\frac{\varepsilon}{2}} \nabla a^{\ell}\right\|_{\widetilde{L}_{T}^{\infty}\left(\dot{B}_{p, 1}^{\frac{d}{p}}\right)} \lesssim \mathcal{D}_{p}^{2}(T), \\
&\left\|t^{\alpha} k(a) \nabla a^{h}\right\|_{\widetilde{L}_{T}^{\infty}\left(\dot{B}_{p, 1}^{\frac{d}{p}-1}\right)}^{h} \lesssim\|a\|_{\widetilde{L}_{T}^{\infty}\left(\dot{B}_{p, 1}^{\frac{d}{p}}\right)}\left\|t^{\alpha} \nabla a^{h}\right\|_{\widetilde{L}_{T}^{\infty}\left(\dot{B}_{p, 1}^{\frac{d}{p}-1}\right)} \lesssim \mathcal{E}_{p}(T) \mathcal{D}_{p}(T) .
\end{aligned}
$$

The term $g_{3}(a, u)$ is of the type $H(a) \nabla^{2} u$ with $H(0)=0$, and the term $g_{4}(a, u)$ is of the type $\nabla K(a) \otimes \nabla u$ with $K(0)=0$. Consequently, we derive that thanks to Propositions 3.2 and 3.4 as well as (4.41)-(4.42),

$$
\begin{aligned}
& \left\|t^{\alpha} H(a) \nabla^{2} u^{\ell}\right\|_{\widetilde{L}_{T}^{\infty}\left(\dot{B}_{p, 1}^{\frac{d}{p}-1}\right)}^{h}+\left\|t^{\alpha} \nabla K(a) \otimes \nabla u^{\ell}\right\|_{\widetilde{L}_{T}^{\infty}\left(\dot{B}_{p, 1}^{\frac{d}{p}-1}\right)}^{h} \\
\lesssim & \left\|t^{\frac{\sigma_{1}}{2}+\frac{d}{4}-\frac{\varepsilon}{2}} a\right\|_{\widetilde{L}_{T}^{\infty}\left(\dot{B}_{p, 1}^{\frac{d}{p}}\right)}\left\|t^{\frac{\sigma_{1}}{2}+\frac{d}{4}+\frac{1}{2}-\frac{\varepsilon}{2}} \nabla u^{\ell}\right\|_{\widetilde{L}_{T}^{\infty}\left(\dot{B}_{p, 1}^{\frac{d}{p}}\right)} \lesssim \mathcal{D}_{p}^{2}(T), \\
& \left\|t^{\alpha} H(a) \nabla^{2} u^{h}\right\|_{\widetilde{L}_{T}^{\infty}\left(\dot{B}_{p, 1}^{\frac{d}{p}-1}\right)}^{h}+\left\|t^{\alpha} \nabla K(a) \otimes \nabla u^{h}\right\|_{\widetilde{L}_{T}^{\infty}\left(\dot{B}_{p, 1}^{\frac{d}{p}-1}\right)}^{h} \\
\lesssim & \|a\|_{\widetilde{L}_{T}^{\infty}\left(\dot{B}_{p, 1}^{\frac{d}{p}}\right)}\left\|t^{\alpha} \nabla u^{h}\right\|_{\widetilde{L}_{T}^{\infty}\left(\dot{B}_{p, 1}^{\frac{d}{p}}\right)} \lesssim \mathcal{E}_{p}(T) \mathcal{D}_{p}(T) .
\end{aligned}
$$

Therefore, we obtain

$$
\left\|t^{\alpha} g(a, u)\right\|_{\widetilde{L}_{T}^{\infty}\left(\dot{B}_{p, 1}^{\frac{d}{p}-1}\right)}^{h} \lesssim \mathcal{D}_{p}^{2}(T)+\mathcal{E}_{p}(T) \mathcal{D}_{p}(T) .
$$

Furthermore, reverting to (4.40), we end up with

$$
\sum_{j \geq j_{0}-1} 2^{j\left(\frac{d}{p}-1\right)} \sup _{1 \leq t \leq T} t^{\alpha}\left(Z_{j}^{1}(t)+Z_{j}^{2}(t)\right) \lesssim \mathcal{D}_{p}^{2}(T)+\mathcal{E}_{p}(T) \mathcal{D}_{p}(T) .
$$


The term $Z_{j}^{3}$ is similar to $g_{3}(a, u)$ and $g_{4}(a, u)$ of $g$. Indeed, we have

$$
\begin{aligned}
\sum_{j \geq j_{0}-1} 2^{j\left(\frac{d}{p}-1\right)} \sup _{1 \leq t \leq T} t^{\alpha} Z_{j}^{3}(t) \lesssim & \left\|t^{\frac{\sigma_{1}}{2}+\frac{d}{4}-\frac{\varepsilon}{2}} a\right\|_{\widetilde{L}_{T}^{\infty}\left(\dot{B}_{p, 1}^{\frac{d}{p}}\right)}\left\|t^{\frac{\sigma_{1}}{2}+\frac{d}{4}+\frac{1}{2}-\frac{\varepsilon}{2}} \operatorname{div} u^{\ell}\right\|_{\widetilde{L}_{T}^{\infty}\left(\dot{B}_{p, 1}^{\frac{d}{p}}\right)} \\
& +\|a\|_{\widetilde{L}_{T}^{\infty}\left(\dot{B}_{p, 1}^{\frac{d}{p}}\right)}\left\|t^{\alpha} \operatorname{div} u^{h}\right\|_{\widetilde{L}_{T}^{\infty}\left(\dot{B}_{p, 1}^{\frac{d}{p}}\right)} \\
\lesssim & \mathcal{D}_{p}^{2}(T)+\mathcal{E}_{p}(T) \mathcal{D}_{p}(T) .
\end{aligned}
$$

For the term $Z_{j}^{4}$, we employ a small modification of the commutator estimate as in Proposition 3.5 (just include $t^{\alpha}$ in the definition of the commutator, follow the proof treating the time variable as a parameter, and take the supremum on $[0, T]$ at the end) and obtain

$$
\begin{aligned}
\sum_{j \geq j_{0}-1} 2^{j\left(\frac{d}{p}-1\right)} \sup _{0 \leq t \leq T} t^{\alpha}\left\|R_{j}(t)\right\|_{L^{p}} \lesssim & \left\|t^{\frac{\sigma_{1}}{2}+\frac{d}{4}+\frac{1}{2}-\frac{\varepsilon}{2}} \nabla u^{\ell}\right\|_{\widetilde{L}_{T}^{\infty}\left(\dot{B}_{p, 1}^{\frac{d}{p}}\right)}\left\|t^{\frac{\sigma_{1}}{2}+\frac{d}{4}-\frac{\varepsilon}{2}} a\right\|_{\widetilde{L}_{T}^{\infty}\left(\dot{B}_{p, 1}^{\frac{d}{p}}\right)} \\
& +\left\|t^{\alpha} \nabla u^{h}\right\|_{\widetilde{L}_{T}^{\infty}\left(\dot{B}_{p, 1}^{\frac{d}{p}}\right)}\|a\|_{\widetilde{L}_{T}^{\infty}\left(\dot{B}_{p, 1}^{\frac{d}{p}}\right)} \cdot
\end{aligned}
$$

Hence, taking advantage of (4.41) and (4.42) gives

$$
\sum_{j \geq j_{0}-1} 2^{j\left(\frac{d}{p}-1\right)} \sup _{0 \leq t \leq T} t^{\alpha}\left\|R_{j}(t)\right\|_{L^{p}} \lesssim \mathcal{D}_{p}^{2}(T)+\mathcal{E}_{p}(T) \mathcal{D}_{p}(T) .
$$

The term $Z_{j}^{5}$ is clearly bounded by the r.h.s. of (4.43). Putting all the above estimates together, we thus have

$$
\sum_{j \geq j_{0}-1} 2^{j\left(\frac{d}{p}-1\right)} \sup _{1 \leq t \leq T} t^{\alpha} Z_{j}(t) \lesssim \mathcal{E}_{p}(T) \mathcal{D}_{p}(T)+\mathcal{D}_{p}^{2}(T) .
$$

Plugging (4.44) in (4.39), and remembering (4.35), (4.37) and (4.38), we eventually conclude that (4.34). Hence, the proof of Proposition 4.2 is complete.

4.3. Third step: Decay and gain of regularity for the high frequency of $u$. In the last step, our aim is to bound the third norm in the functional $\mathcal{D}_{p}(t)$ and close the time-weighted energy inequality.

Proposition 4.3. If $p$ satisfies (2.2), then it holds that for all $t \geq 0$,

$$
\left\|\tau^{\alpha} \nabla u\right\|_{\widetilde{L}_{t}^{\infty}\left(\dot{B}_{p, 1}^{\frac{d}{p}}\right)}^{h} \lesssim\left\|\left(\nabla a_{0}, u_{0}\right)\right\|_{\dot{B}_{p, 1}^{\frac{d}{p}-1}}^{h}+\mathcal{E}_{p}^{2}(t)+\mathcal{D}_{p}^{2}(t)
$$

with $\alpha=\sigma_{1}+\frac{d}{2}+\frac{1}{2}-\varepsilon$ for sufficiently small $\varepsilon>0$, where $\mathcal{E}_{p}(t)$ and $\mathcal{D}_{p}(t)$ are defined by (4.1) and (2.6), respectively.

Proof. The proof lies in a new observation, which enables us to obtain more decay for the improved regularity of velocity. Precisely, it follows from the velocity equation in (2.1) that

$$
\partial_{t} u-\mathcal{A} u=g-\nabla a .
$$

To obtain desired estimates, we reformulate (4.46) as follows

$$
\left\{\begin{array}{l}
\partial_{t}\left(t^{\alpha} \mathcal{A} u\right)-\mathcal{A}\left(t^{\alpha} \mathcal{A} u\right)=t^{\alpha} \mathcal{A} g+\alpha t^{\alpha-1} \mathcal{A} u-t^{\alpha} \mathcal{A} \nabla a \\
\left.t^{\alpha} \mathcal{A} u\right|_{t=0}=0
\end{array}\right.
$$


Taking advantage of the smoothing property for Lamé semi-group in Remark 3.2, we thus get

$$
\left\|\tau^{\alpha} \nabla^{2} u\right\|_{\widetilde{L}_{t}^{\infty}\left(\dot{B}_{p, 1}^{\frac{d}{p}-1}\right)}^{h} \lesssim\left\|\tau^{\alpha} g\right\|_{\widetilde{L}_{t}^{\infty}\left(\dot{B}_{p, 1}^{\frac{d}{p}-1}\right)}^{h}+\left\|\tau^{\alpha-1} u\right\|_{\widetilde{L}_{t}^{\infty}\left(\dot{B}_{p, 1}^{\frac{d}{p}-1}\right)}^{h}+\left\|\tau^{\alpha} \nabla a\right\|_{\widetilde{L}_{t}^{\infty}\left(\dot{B}_{p, 1}^{\frac{d}{p}-1}\right)}^{h} .
$$

Due to the fact $\alpha>1$, we see that

$$
\left\|\tau^{\alpha-1} u\right\|_{\widetilde{L}_{t}^{\infty}\left(\dot{B}_{p, 1}^{\frac{d}{p}-1}\right)}^{h} \lesssim\left\|\langle\tau\rangle^{\alpha} u\right\|_{\widetilde{L}_{t}^{\infty}\left(\dot{B}_{p, 1}^{p}-1\right.}^{h}, \quad\left\|\tau^{\alpha} \nabla a\right\|_{\widetilde{L}_{t}^{\infty}\left(\dot{B}_{p, 1}^{\frac{d}{p}-1}\right)}^{h} \lesssim\left\|\langle\tau\rangle^{\alpha} a\right\|_{\widetilde{L}_{t}^{\infty}\left(\dot{B}_{p, 1}^{\frac{d}{p}}\right)}^{h} .
$$

Furthermore, we conclude that

$$
\left\|\tau^{\alpha} \nabla u\right\|_{\widetilde{L}_{t}^{\infty}\left(\dot{B}_{p, 1}^{\frac{d}{p}}\right)}^{h} \lesssim\left\|\tau^{\alpha} g\right\|_{\widetilde{L}_{t}^{\infty}\left(\dot{B}_{p, 1}^{\frac{d}{p}-1}\right)}^{h}+\left\|\langle\tau\rangle^{\alpha}(\nabla a, u)\right\|_{\widetilde{L}_{t}^{\infty}\left(\dot{B}_{p, 1}^{\frac{d}{p}-1}\right)}^{h} .
$$

In light of (4.34), the second norm can be bounded by

$$
\left\|\left(\nabla a_{0}, u_{0}\right)\right\|_{\dot{B}_{p, 1}^{\frac{d}{p}-1}}^{h}+\mathcal{E}_{p}^{2}(t)+\mathcal{D}_{p}^{2}(t) .
$$

Bounding the norm $\left\|\tau^{\alpha} g\right\|_{\widetilde{L}_{t}^{\infty}\left(\dot{B}_{p, 1}^{\frac{d}{p}-1}\right)}^{h}$ is exactly same as the second step, and one thus arrive at (4.45) readily.

Finally, adding up (4.45) to (4.33) and (4.34) yields for all $T \geq 0$,

$$
\mathcal{D}_{p}(T) \lesssim \mathcal{D}_{p, 0}+\left\|\left(\nabla a_{0}, u_{0}\right)\right\|_{\dot{B}_{p, 1}^{\frac{d}{p}-1}}^{h}+\mathcal{E}_{p}^{2}(T)+\mathcal{D}_{p}^{2}(T) .
$$

The global existence result (see Theorem 1.1 in [10]) ensures that $\mathcal{E}_{p}(t) \lesssim \mathcal{E}_{p, 0} \ll 1$ and as

$$
\left\|\left(a_{0}, u_{0}\right)\right\|_{\dot{B}_{2,1}^{2}-1}^{\ell} \lesssim \mathcal{D}_{p, 0} \ll 1,
$$

one can conclude that (2.5) is satisfied for all time if $\mathcal{D}_{p, 0}$ and $\mathcal{E}_{p, 0}$ are small enough. This completes the proof of Theorem 2.1.

\section{Appendix}

We end the paper with some heuristics concerning the optimality of the regularity and decay exponents in the definition of $\mathcal{D}_{p}(t)$. Let us first explain why the regularity exponent $\sigma$ in the first term $\mathcal{D}_{p}(t)$ has to satisfy $\sigma \leq \frac{d}{2}+1$. The general fact (based on Inequality (4.2)) that we used repeatedly is that the time decay exponent $\delta$ for $\|(f, g)\|_{\dot{B}_{2, \infty}^{-\sigma_{1}}}$ must satisfy $\delta \geq \frac{\sigma_{1}+\sigma}{2}$. Now, if we look at the term $a^{\ell} \operatorname{div} u^{\ell}$, then a necessary condition for having

$$
\left\|a^{\ell} \operatorname{div} u^{\ell}\right\|_{\dot{B}_{2, \infty}^{-\sigma_{1}}}^{\ell} \lesssim\left\|a^{\ell}\right\|_{\dot{B}_{2, \infty}^{\sigma_{2}}}\left\|\operatorname{div} u^{\ell}\right\|_{\dot{B}_{2, \infty}^{\sigma_{3}}}
$$

is that $\sigma_{2}+\sigma_{3} \leq \frac{d}{2}-\sigma_{1}$. As the decay exponent of the right-hand side is $\sigma_{1}+\frac{\sigma_{2}+\sigma_{3}+1}{2}$, we deduce that $\delta \leq \frac{d}{4}+\frac{\sigma_{1}}{2}+\frac{1}{2}$. Hence we must have $\sigma \leq \frac{d}{2}+1$.

To see that the decay rate for the second term in $\mathcal{D}_{p}(t)$ cannot be more than $\sigma_{1}+\frac{d}{2}+\frac{1}{2}$, one can observe that, owing to $\sigma \leq \frac{d}{2}+1$, the term $a^{\ell} \nabla a^{\ell}$ (which at most has the same regularity as $\left.\nabla a^{\ell}\right)$ cannot be estimated in a space with higher regularity than $\dot{B}_{2,1}^{\frac{d}{2}}$. As the corresponding estimate reads

$$
\left\|a^{\ell} \nabla a^{\ell}\right\|_{\dot{B}_{2,1}^{\frac{d}{2}}} \lesssim\left\|a^{\ell}\right\|_{\dot{B}_{2,1}^{\frac{d}{2}}}\left\|\nabla a^{\ell}\right\|_{\dot{B}_{2,1}^{\frac{d}{2}}}
$$


it follows from the first term in $\mathcal{D}_{p}(t)$ that the right-hand side has decay exponent $\sigma_{1}+\frac{d}{2}+\frac{1}{2} \cdot$ A similar argument shows that the decay rate for the third term in $\mathcal{D}_{p}(t)$ is optimal.

\section{ACKNOWLEDGMENTS}

The author is partially supported by the National Natural Science Foundation of China (11471158) and the Fundamental Research Funds for the Central Universities (NE2015005). Last but not least, he would like to thank Dr. Weixuan Shi for some communications.

\section{REFERENCES}

[1] H. Bahouri, J. Y. Chemin and R. Danchin: Fourier Analysis and Nonlinear Partial Differential Equations, Grundlehren der mathematischen Wissenschaften, Vol. 343, Springer, Berlin (2011).

[2] M. Cannone: A generalization of a theorem by Kato on Navier-Stokes equations, Rev. Mat. Iberoamericana, 13, 515-542 (1997).

[3] J. Y. Chemin: Théorèmes d'unicité pour le systèm de Navier-Stokes tridimensionnel, J. Amal. Math., 77, 27-50 (1999).

[4] F. Charve and R. Danchin: A global existence result for the compressible Navier-Stokes equations in the critical $L^{p}$ framework, Arch. Rational Mech. Anal., 198, 233-271 (2010).

[5] J. Y. Chemin and N. Lerner: Flot de champs de vecteurs non lipschitziens et équations de NavierStokes, J. Differ. Equ., 121, 314-328 (1995).

[6] Q. L. Chen, C. X. Miao and Z. F. Zhang: Global well-posedness for compressible Navier-Stokes equations with highly oscillating initial velocity, Comm. Pure Appl. Math., 63, 1173-1224 (2010).

[7] R. Danchin: Global existence in critical spaces for compressible Navier-Stokes equations, Invent. Math., 141, 579-614 (2000).

[8] R. Danchin: Fourier Analysis Methods for the Compressible Navier-Stokes Equations, Handbook of Mathematical Analysis in Mechanics of Viscous Fluids, Y. Giga and A. Novotny editors, Springer International Publishing Switzerland, 2016.

[9] R. Danchin and L. He: The incompressible limit in $L^{p}$ type critical spaces. Math. Ann., 366, 1365-1402 (2016).

[10] R. Danchin and J. Xu: Optimal time-decay estimates for the compressible Navier-Stokes equations in the critical $L^{p}$ framework, Arch. Rational Mech. Anal., 224, 53-90 (2017).

[11] E. Feireisl, A. Novotny and H. Petzeltová, On the existence of globally defined weak solutions to the Navier-Stokes equations, J. Math. Fluid Mech., 3, 358-392, (2001).

[12] W. Fiszdon and W. M. Zajaczkowski, Existence and uniqueness of solutions of the initial boundary value problem for the flow of a baratropic viscous fluid, local in time, Arch. Mech., 35, 497-516 (1983).

[13] H. Fujita and T. Kato: On the Navier-Stokes initial value problem I, Arch. Rational Mech. Anal., 16, 269-315 (1964).

[14] Y. Guo and Y. J. Wang: Decay of dissipative equations and negative sobolev spaces. Comm. Part. Differ. Equ., 37, 2165-2208 (2012).

[15] B. Haspot: Existence of global strong solutions in critical spaces for barotropic viscous fluids, Arch. Rational Mech. Anal., 202, 427-460 (2011).

[16] D. Hoff: Global solutions of the Navier-Stokes equations for multidimensional compressible flow with discontinuous initial data, J. Differ. Equ., 120, 215-254 (1995) .

[17] D. Hoff and K. Zumbrun: Multidimensional diffusion waves for the Navier-Stokes equations of compressible flow, Indiana Univ. Math. J., 44, 604-676 (1995).

[18] X. D. Huang, J. Li and Z. Xin: Global well-posedness of classical solutions with large oscillations and vacuum to the three-dimensional isentropic compressible Navier-Stokes equations, Comm. Pure Appl. Math., 65, 549-585 (2012).

[19] S. Jiang and P. Zhang: Axisymmetric solutions of the 3D Navier-Stokes equations for compressible isentropic fluids, J. Math. Pure Appl., 82, 949-973 (2003).

[20] H. L. Li and T. Zhang: Large time behavior of isentropic compressible Navier-Stokes system in $\mathbb{R}^{3}$, Math. Methods Appl. Sci., 34, 670-682 (2011). 
[21] P. L. Lions, Mathematical topics in fluid mechanics. Vol. 2. Compressible models. Oxford Lecture Series in Mathematics and Its Applications, 10. Clarendon, University Press, New York, 1998.

[22] T. P. Liu and W. K. Wang: The pointwise estimates of diffusion waves for the Navier-Stokes equations in odd multi-dimensions, Commun. Math. Phys. 196, 145-173 (1998).

[23] S. Kawashima: Systems of a hyperbolic-parabolic composite type, with applications to the equations of magnetohydrodynamics, Doctoral Thesis, Kyoto University, 1984.

[24] Y. Kagei and T. Kobayashi: On large time behavior of solutions to the compressible Navier-Stokes equations in the half space in $\mathbb{R}^{3}$, Arch. Rational Mech. Anal., 165, 89-159 (2002).

[25] Y. Kagei and T. Kobayashi: Asymptotic behavior of solutions of the compressible Navier-Stokes equations on the half space. Arch. Rational Mech. Anal., 177, 231-330 (2005).

[26] T. Kobayashi: Some estimates of solutions for the equations of motion of compressible viscous fluid in an exterior domain in $\mathbb{R}^{3}$, J. Differ. Equ., 184, 587-619 (2002).

[27] T. Kobayashi and Y. Shibata: Decay estimates of solutions for the equations of motion of compressible viscous and heat-conductive gases in an exterior domain of $\mathbb{R}^{3}$, Comm. Math. Phys., 200, 621-659 (1999).

[28] H. Kozono and M. Yamazaki: Semilinear heat equations and the Navier-Stokes equations with distributions in new function spaces as initial data, Comm. Part. Differ. Equ., 19, 959-1014 (1994).

[29] A. Matsumura and T.Nishida: The initial value problem for the equation of motion of compressible viscous and heat-conductive fluids, Proc. Jpn. Acad. Ser-A, 55, 337-342 (1979).

[30] A. Matsumura and T. Nishida: The initial value problem for the equations of motion of viscous and heat-conductive gases, J. Math. Kyoto Univ., 20, 67-104 (1980).

[31] J. Nash: Le problme de Cauchy pour les équations différentielles d'un fluide général, Bull. Soc. Math. France, 90, 487-497 (1962).

[32] M. Okita: Optimal decay rate for strong solutions in critical spaces to the compressible NavierStokes equations, J. Differ. Equ., 257, 3850-3867 (2014).

[33] G. Ponce: Global existence of small solution to a class of nonlinear evolution equations, Nonlinear Anal. TMA, 9, 339-418 (1985).

[34] J. Serrin: On the uniqueness of compressible fluid motion, Arch. Rational. Mech. Anal., 3, 271-288 (1959).

[35] V. Sohinger and R. M. Strain: The Boltzmann equation, Besov spaces, and optimal time decay rates in $\mathbb{R}_{x}^{n}$, Adv. Math., 261, 274-332 (2014).

[36] V. A. Solonnikov, Estimates of soutions to a nonstationnary Navier-Stokes system, Zap. Nauchn. Semin. LOMI, 38, 153-231 (1973).

[37] A. Valli, An existence theorem for compressible viscous fluids, ANN. Mat. Pura Appl., 130, 197213 (1982); 132, 399-400 (1982).

[38] Z. Xin, Blowup of smooth solutions to the compressible Navier-Stokes equation with compact density, Comm. Pure Appl. Math., 51, 229-240 (1998).

[39] J. Xu and S. Kawashima: Frequency-localization Duhamel principle and its application to the optimal decay of dissipative systems in low dimensions, J. Differ. Equ., 261, 2670-2701 (2016).

[40] J. Xu and S. Kawashima: The optimal decay estimates on the framework of Besov spaces for generally dissipative systems, Arch. Rational Mech. Anal., 218, 275-315 (2015).

[41] Y. Zeng: $L^{1}$ Asymptotic behavior of compressible isentropic viscous 1-D flow, Comm. Pure Appl. Math., 47, 1053-1082 (1994).

Department of Mathematics, Nanjing University of Aeronautics and Astronautics, NANJING 211106, P.R.ChINA,

E-mail address: jiangxu_79math@yahoo.com 